\title{
Forced unfolding of protein-inspired single-chain
}

$7 \quad *$ Correspondence: aalexand@mit.edu

8 KEYWORDS. Random heteropolymer (RHP), bioinspired, compactification, single-molecule

9 mechanics, unfolding, topology, atomistic molecular dynamics, PEGylation

\section{For Table of Contents use only}

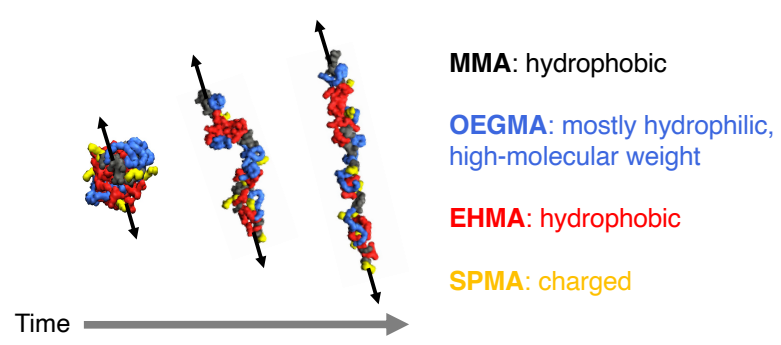




\section{ABSTRACT}

14 Synthetic random heteropolymers (RHPs) with high chemical heterogeneity can self-assemble into

15 single-chain nanoparticles that exhibit features reminiscent of natural proteins, such as topological

16 polymorphism. Using all-atom molecular dynamics simulations, this work investigates the

17 structure and single-chain mechanical unfolding of a library of four-component RHPs in water,

18 studying the effects of sequence, composition, configuration, and molecular weight. Results show

19 that compactified RHPs can have highly dynamic unfolding behaviors which are dominated by

20 complex side-chain interactions and prove markedly different from their homopolymer

21 counterparts. For a given sequence and conformation, an RHP's backbone topology can strongly

22 impact its unfolding response, hinting at the importance of topological design in the nanoscale

23 mechanics of heteropolymers. In addition, we identify enthalpically-driven reconfiguration upon

24 unfolding, observing a solvent-shielding protection mechanism similar to protein stabilization by

25 PEGylation. This work provides the first computational evidence for the force-induced unfolding

26 of protein-inspired multicomponent heteropolymers.

\section{INTRODUCTION}

29 The need to study single molecules stemmed from the desire to develop a more thorough

30 understanding of the biophysics of proteins or nucleic acids when subjected to a form of stress.

31 Compared to chemical or thermal stimuli, mechanical force serves as a distinct and orthogonal

32 strategy to study single-molecule mechanics. ${ }^{1}$ For folded proteins, highly heterogeneous

33 mechanical responses are possible and proteins can undertake a range of mechanical (load-bearing

34 or mechanosensing) and non-mechanical functions. One of the protein systems most commonly

35 studied for its mechanical behavior is titin, a muscle protein exhibiting high elasticity. Force- 
36 induced unfolding of a titin molecule reveals that its modular domains extend sequentially and

37 independently, where each domain unfolding event gives rise to one force peak (or rupture force),

38 attributable to the severing of a specific set of interstrand hydrogen bonds..$^{2-4}$ Titin, therefore, gives

39 rise to sawtooth patterns in force curves, which are also seen for other proteins for which secondary

40 and/or tertiary structure lead to defined domains, such as spectrin and ankyrin. ${ }^{3,5,6}$ More generally,

41 force-extension profiles for protein unfolding have distinguishable features arising from the

42 sequence-defined structural interactions between amino acid side groups, such as the separation of

$43 \beta$-sheets and the uncoiling of $\alpha$-helices. It naturally follows that, through examination of the

44 unfolding behavior, structural information can be gained. Through understanding the relative

45 forces required for dissociation of various portions of the proteins, insights into how the molecules

46 may respond when disrupted by an external force - be it mechanical, chemical, or thermal - are

47 provided.

48 While unfolding has been studied extensively for proteins and other natural biomacromolecules,

49 there exist fewer studies on the single-chain mechanics of synthetic macromolecules with

50 significant chemical complexity. Unfolding of hydrophobic homopolymer globules is well-

51 studied; ${ }^{7-9}$ though, more complex collapsed synthetic polymers are less well characterized. A

52 subset of these complex macromolecules, which have been examined somewhat more thoroughly,

53 assemble through the incorporation of modularity and orthogonal chemistries. ${ }^{10-12}$ Chung et al.

54 designed a modular polymer mimicking titin, exploiting the ability of the constituent monomer 2-

55 ureido-4-[1H]-pyrimidinone (UPy) to self-dimerize through hydrogen bonding. ${ }^{10}$ Hosono et al.

56 reported the mechanical unfolding of a similarly designed single-chain nanoparticle (SCNP),

57 where the pendants within the chain can intramolecularly crosslink, leading to supramolecular self-

58 assembly or self-collapse. ${ }^{11}$ As a result of such modularity, these two biomimetic polymers display 
59 characteristic sawtooth patterns in their respective force-extension profiles and stepwise unfolding

60 pathways. Metal- $\pi$ coordination chemistry has also been exploited for creating self-folding single-

61 chains in which rupturing of transient linkages can dissipate energy. ${ }^{12}$ These examples rely on

62 specific, directional interactions. Fewer studies investigate the unfolding of globularly structured

63 SCNPs that assemble due to nonspecific interactions. Geissler and Shakhnovich were the first to

64 lay out an analytical treatment for the mechanical unfolding of general random heteropolymers

65 (RHPs). ${ }^{13,14}$ They proposed that, during heteropolymer unfolding, there will likely have a pearl-

66 necklace-shaped intermediate. The existence of this morphology can be ascribed to solvation

67 effects, where hydrophilic regions are prone to extend upon unfolding whilst hydrophobic regions

68 remain collapsed and compact to minimize solvent exposure, forming "pearls". We expect that the

69 stability of pearl-necklace morphologies will be dependent on the exact sequence. For instance, a

70 heteropolymer where hydrophobic clusters are periodically spaced within the chain will likely

71 experience this necklace-like intermediate compared to one that is completely random. While

72 insights can be gained from these studies, they lack chemical detail which we have previously

73 demonstrated to be vital to understanding specific random heteropolymer assembly. ${ }^{15}$

74 Of the existing reports on the forced unfolding of macromolecules, most work is experimentally

75 enabled by a suite of single-molecule force spectroscopy techniques including atomic force

76 microscopy, optical tweezers, and magnetic tweezers. ${ }^{16,17}$ However, these techniques fail to help

77 visualize the purported unfolding events. An area for exploration is in situ imaging of single

78 molecules during mechanical unfolding for multimodal analysis. Both single-molecule

79 fluorescence imaging and in situ liquid-cell electron microscopy may be possible candidates,

80 though their developments are only in their infancy. ${ }^{18,19}$ In silico methods modeling single-

81 molecule nanomechanics are therefore an attractive alternative, capable of providing mechanistic 
82 insights. Common computational methods to study single-molecule mechanical response include

83 all-atom steered molecular dynamics (SMD) $)^{2,20,21}$, coarse-grained Brownian dynamics ${ }^{7}$, and Monte

84 Carlo simulations ${ }^{22}$. Among these, all-atom SMD is particularly favored for studying the forced

85 unfolding of chemically heterogenous biomolecules due to the atomistic resolution that proves

86 essential to capturing their conformational flexibility and diversity. Atomistic modeling also

87 explicitly includes polar interactions with water molecules, which is particularly useful in

88 modeling the unique amphiphilic behavior of polyethylene glycol (PEG). ${ }^{23,24}$ One evident

89 limitation of atomistic SMD and similar computational techniques is the difficulty to access micro-

90 to millisecond timescales due to a correspondingly high computational cost. As a result, the typical

91 pulling speed employed in SMD lies in the range of $10-100 \AA \mathrm{ns}^{-1}$ (Table S1), which is six to

92 seven magnitudes faster compared to those used in experimental methods. ${ }^{20-22,25-31}$ Due to the

93 orders-of-magnitude difference in pulling velocities, SMD results often overestimate force peak

94 values compared to empirical values. In spite of this, it is still common to correlate experimental

95 findings with simulations in order to gain mechanistic insights into the unfolding events and

96 elucidate unfolding pathway(s), and there is typically a satisfactory qualitative agreement between

97 simulations and experiments..$^{21,32}$

98 Overall, single-molecule mechanics is of paramount importance for understanding the internal

99 structure and response to external forces of polymeric chains. Using a recently reported RHP

100 system as an example ${ }^{33}$, this work characterizes the single-chain mechanical response of a highly

101 chemically heterogeneous polymer system. $\mathrm{Xu}$ and colleagues took inspiration from natural

102 proteins and rationally designed methacrylate-based statistical RHPs, which serve as a novel class

103 of biomimetic materials. ${ }^{33}$ These four-component RHPs incorporate methyl methacrylate (MMA),

104 oligo(ethylene glycol) methacrylate (OEGMA), 2-ethylhexyl methacrylate (EHMA), and 3- 
105 sulfopropyl methacrylate (SPMA) (Figure 1A). The monomer selections are intended to leverage

106 varied amphiphilicity and polarity to recapitulate the heterogeneity of native protein chains and

107 resulting surfaces. ${ }^{15}$ Such a design differs from many past SCNPs, which rely on intramolecular

108 crosslinking strategies, and more closely resembles the self-assembly of natural

109 biomacromolecules. ${ }^{10-12}$ The RHPs can mimic protein functions and interface favorably with

110 proteins, finding applications as synthetic alternatives to molecular chaperones to stabilize proteins

111 in non-native environments ${ }^{33,34}$, as mimics of transmembrane proteins to facilitate selective proton

112 transport ${ }^{35}$, and as enzyme protectants to facilitate the degradation of commodity plastics ${ }^{36}$.

113 Atomistic modeling of these RHPs in water revealed that while the RHP structures are not

114 sequence-defined, some structural motifs emerge in their collapsed form and a variety of

115 assemblies are possible. ${ }^{15}$ We also showed that the RHPs possess minimal backbone mobility in

116 water; however, experiments have demonstrated that the RHPs can interact with other

117 biomacromolecules and small molecules, suggesting that external stimuli could provide the driving

118 force to at least partially unfold portions of the molecules. Herein, we perform all-atom MD

119 simulations to characterize RHPs with degrees of polymerization of 20 and 50 (referred to herein

120 as 20mers and 50mers, respectively) in water. Sequences of various compositions and

121 arrangements of the four methacrylate-based components are investigated (Figure 1A) using SMD

122 to study their unfolding (Figure 1B). To the best of our knowledge, no prior work has been

123 performed to investigate the unfolding behavior of synthetic heteropolymers as chemically

124 complex as the four-component amphiphilic RHPs presented here. Aforementioned theoretical

125 treatments of heteropolymers often neglect atomistic details and cannot accurately capture the

126 conformational complexity of chemically heterogeneous polymers. ${ }^{13,14,37}$ Thus, our investigation

127 of this bioinspired RHP system not only adds a different chemistry to the current portfolio of forced 
128 unfolding of synthetic heteropolymers, but also affords a library of polymer sequences for 129 investigation. Moreover, by understanding how the RHP responds to a tensile force stimulus, we 130 can appreciate what would likely be required for backbone remodeling to take place and gain 131 insights into internal structural dynamics and stability of the RHPs in water.

A

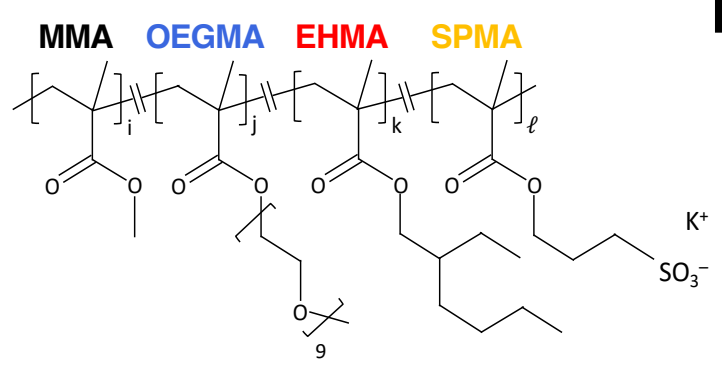

B
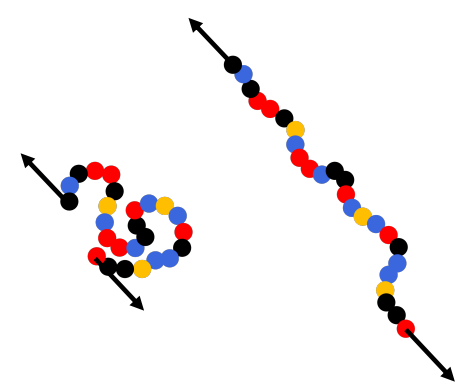

134 Figure 1. Protein-inspired RHP studied in this work. (A) RHP chemical structure. Monomers

135 are color-coded as follows: MMA in black, OEGMA in blue, EHMA in red, and SPMA in yellow.

(B) Schematic showing the forced unfolding of an RHP from its collapsed state.

\section{RESULTS \& DISCUSSION}

\section{RHP chain compactification}

140 The self-assembly of explicitly solvated single-chain heteropolymers is studied, with sequence 141 schematics of all 20mer and 50mer RHPs investigated in this work provided in Figure S1.

142 Similarly to 100 mer RHPs of the same chemistry ${ }^{15}$, individual 20 mer and 50mer RHP sequences 143 can self-assemble into multiple conformational states. For a given chain, 10 annealing cycles lead 144 to the sampling of 10 distinct conformers, each with unique topological organization in the 145 backbone (Figures S2 and S3). Such topological heterogeneity has also been observed in certain 146 intrinsically disordered proteins as well as synthetic SCNPs of similar sizes, alluding to the rich 
147 conformational energy landscape of these comparable systems. ${ }^{38,39}$ Standard deviation of the 148 absolute value of backbone dihedral angles is used as a measure for backbone mobility over the 149 relevant timescale. By this measure, the ten conformational states sampled are believed to be 150 metastable in nature as the backbones minimally change, as illustrated by the small standard 151 deviation of ldihedral anglesl over $40 \mathrm{~ns}$ for the equilibrated system in water (Figure 2A). For both 152 20mers and 50mers, the ends of the RHPs are, as expected by the configurational entropy of linear 153 polymer chains, more mobile than the middle.

154 Mobility comparisons between RHPs of different molecular weights show that both the middle 155 and end segments of the 50mers reconfigure less than their counterparts in the shorter polymers in 156 unbiased MD simulations. From this trend, we can imply that the longer polymers are more 157 compact, impeding backbone rearrangement. This is confirmed by Figure 2B, whereby an analog 158 for density (polymer mass divided by the radius of gyration, $\mathrm{R}_{\mathrm{g}}$, cubed) generally increases with 159 molecular weight. One notable exception is sequence 19, which shows a decrease in density when 160 comparing its 20 and 50mer lengths. This result stems from the anion-anion repulsion of the SPMA 161 monomers, of which sequence 19 has the highest proportion investigated in this work, leading to 162 polyelectrolyte-like behavior. We would not expect an RHP with a high negative charge to 163 compactify into a globular morphology; instead, an amphiphilic polymer with a high net charge 164 would adopt a more extended conformation, giving rise to a lower density. ${ }^{40,41}$ The typical RHP 165 trend, however, shows compactification as well as a narrowing of the range of densities between 166 sequence conformations as molecular weight increases. The narrower range of density values 167 stems from the greater similarity in $\mathrm{R}_{\mathrm{g}}$ values for 50 mers, as despite different backbone topologies, 168 configurations all led to compact globules. For 20mers, some sequence conformations formed 169 denser assemblies while others remained extended, indicating a stochastic compactification with 
170 close energetic competition between the entropic cost of limiting mobility in a compact globule

171 and the enthalpic surface energy penalty of exposing hydrophobic monomers to the aqueous

172 environment. As smaller oligomers, the RHPs will be soluble, because even MMA, one of the

173 more hydrophobic monomers in our polymer, is soluble at extremely low degrees of

174 polymerization..$^{42}$ Therefore, based on the simulation results, most compositions of RHPs with

175 degree of polymerization of 20 appear to be near the energetic cliff for compact globule formation,

176 while 50mers in the same windows are nearly all compact and more uniform in density.

177

A

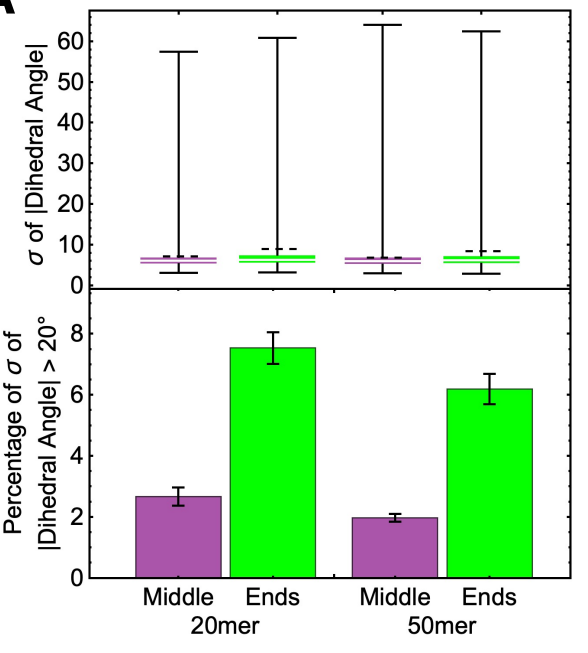

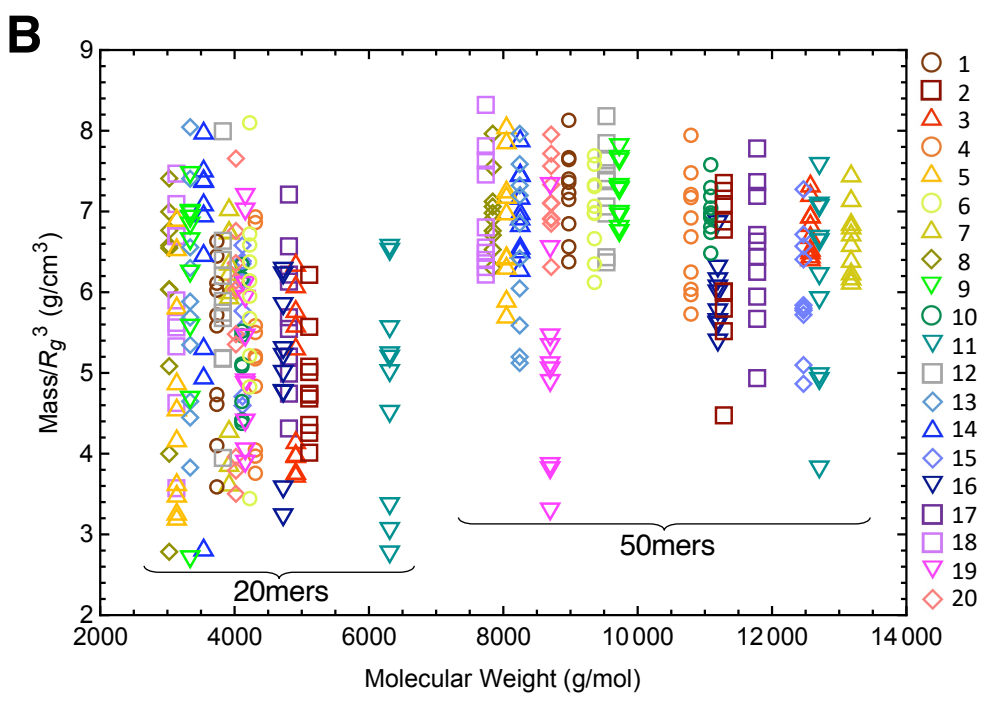

179 Figure 2. RHP chain mobility and its compactification. (A) (Top) Box-and-whiskers plot of the

180 raw standard deviation $\sigma$ of Idihedral anglel for each conformation from the $40 \mathrm{~ns}$ of equilibration,

181 for all 20mer and 50mer RHP sequences and conformations, respectively. Dashed lines represent

182 the mean. The "ends" refer to the 8 dihedral angles from the 5 monomers on each end (thus in total

18316 dihedrals) regardless of the RHP length; the remaining dihedrals are in the "middle" of the

184 RHP. (Bottom) Percentage of $\sigma$ of Idihedral anglel values greater than $20^{\circ}$ for RHP ends vs. middle

185 over the $40 \mathrm{~ns}$ of equilibration. Error bars represent standard error around the mean for the 200 
conformations (20 sequences with 10 conformations each). (B) Density analog for all 20mer and

187 50mer sequence conformations versus molecular weight (MW). The density analog in $\mathrm{g} \mathrm{cm}^{-3}$ is

188 calculated as $\mathrm{MW} \times$ Avogadro's number $\mathrm{N}_{\mathrm{A}} / \mathrm{R}_{\mathrm{g}}{ }^{3}$, with appropriate unit conversions. RHPs 189 generally show compactification as molecular weight increases, with several exceptions explained

190 in text. Corresponding sequence schematics are given in Figure S1.

\section{RHP responses to force-induced unfolding}

193 For a selection of polymer sequences, five independent replicates were studied under an applied

194 tensile force to induce unfolding at a constant rate of $1 \AA \mathrm{ns}^{-1}$. To ensure a sufficiently slow pulling 195 rate for our RHP system, we perform in silico stress-relaxation experiments on one 50mer RHP, 196 sequence 12. Snapshots during the unfolding simulation were extracted every $10 \mathrm{~ns}$ (i.e., 0 ns, 10 $197 \mathrm{~ns}, 20 \mathrm{~ns}, \ldots 100 \mathrm{~ns}$ ), and each is then stress-relaxed by maintaining the end-to-end distance 198 restraint and allowing the chain to equilibrate for $20 \mathrm{~ns}$ while monitoring for relaxation behaviors 199 (Figure 3A). The backbone dihedrals show only a few changes upon stress relaxation for 200 essentially all unfolding intermediates (Figure 3B and S4), indicating relatively insignificant 201 backbone reconfiguration. In addition, the magnitudes of changes in the dihedral angles for the 202 partially unfolded structures are not far from those for the initial equilibrated structure (Figure 3C, 203 top), and the mobility of the backbone of the structural intermediates remains low (Figure 3C, 204 bottom). This suggests that the initial structural snapshots do not deviate much from their stress205 relaxed states. We also see minimal reconfiguration within the side-chains, which are generally 206 more mobile than the backbone, during the 20-ns stress relaxation. In fact, RHP solvation, which 207 is dominated by side-chain/water interactions, remains nearly constant over the course of the 208 stress-relaxation (Figures 3D and S5), indicating extremely rapid water solvation. Provided this 
209 minimal RHP reconfiguration, the pulling rate employed is sufficiently slow for gaining

210 mechanistic insights into the RHP behavior upon unfolding. Our results show that the RHPs are in

211 a pseudo-equilibrium regime during force-induced unfolding, and the unfolding observed through

212 our procedures is likely a low energy pathway.

213
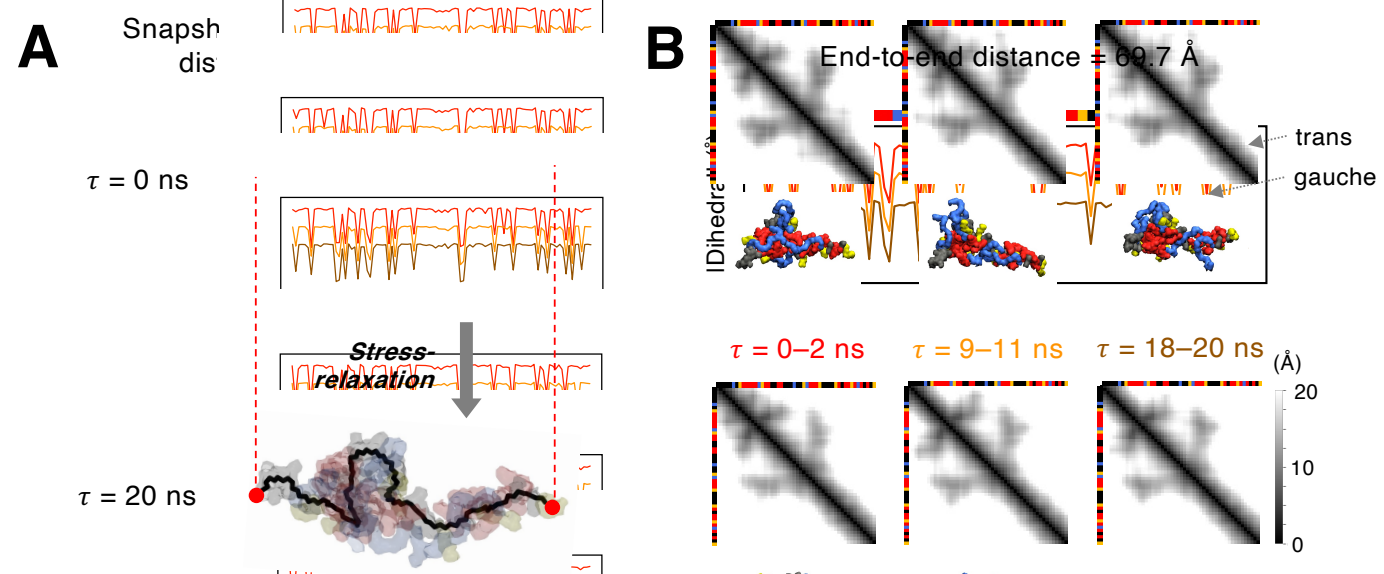

C
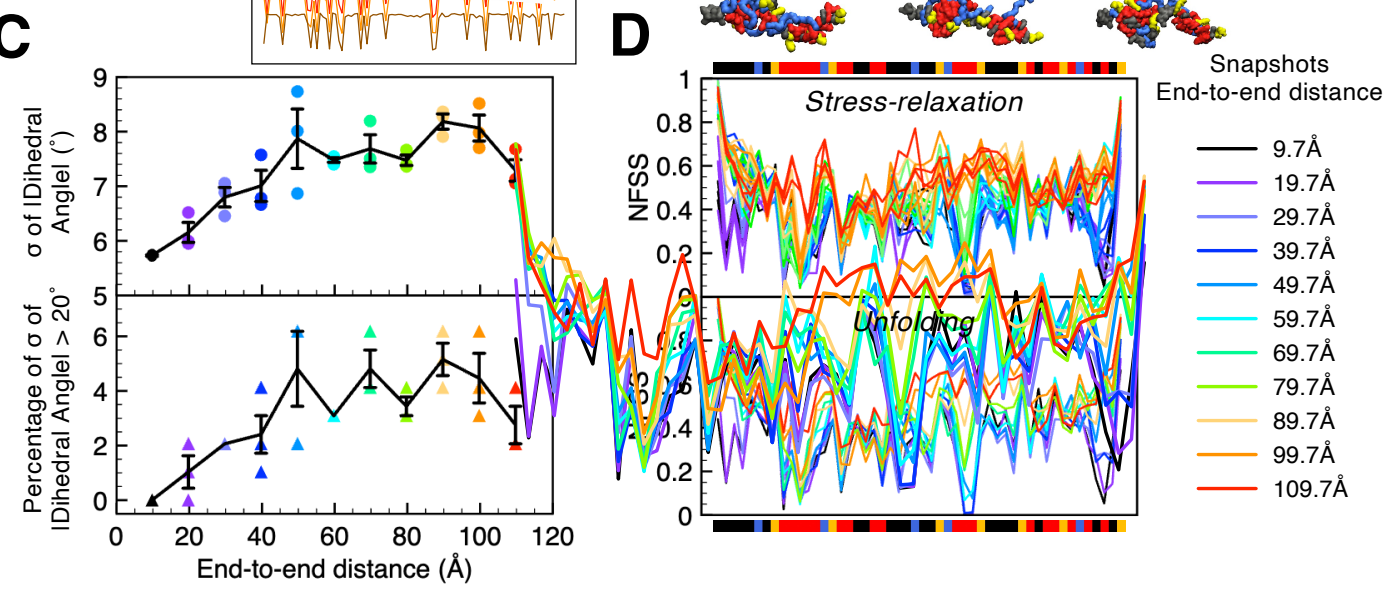

214

215 Figure 3. Insignificant backbone and side-chain remodeling upon RHP stress-relaxation.

216 Results shown here are for 50mer RHP sequence 12 conformation 8. (A) Illustration of the stress-

217 relaxation protocol on an RHP snapshot from unfolding time $t=60 \mathrm{~ns}$. End-to-end distance is

218 maintained constant during the 20-ns stress-relaxation. Note that the stress-relaxation time is

219 represented by $\tau$ in order to differentiate from the unfolding time $t$. (B) Stress-relaxation responses 
220 in the backbone of the snapshot RHP structure from $t=60 \mathrm{~ns}$. Time evolution of dihedral plots,

221 each averaged over 2 ns. Representative time-averaged $C_{\alpha}$-based contact map evolution,

222 confirming insignificant remodeling in the RHP backbone. (C) (Top) Average standard deviation

$223 \sigma$ of the absolute value of all 97 dihedral angles throughout the 20-ns stress-relaxation against

224 different unfolding intermediates. The end-to-end distances correspond to the extracted RHP

225 snapshots from time $t=0 \mathrm{~ns}$ to $t=100 \mathrm{~ns}$ during its unfolding process. Each data point is from one

226 independent stress-relaxation simulation. (Bottom) Average number of dihedrals with a $\sigma$ greater

227 than $20^{\circ}$. Error bars represent standard errors around the mean. (D) Normalized first solvation shell

228 (NFSS) along the sequence for different unfolding intermediates for (top) stress-relaxation and

229 (bottom) mechanical unfolding, showing minimal change in solvation after RHP stress-relaxation.

230 The corresponding time points for each of the end-to-end distances are time-averaged: for stress-

231 relaxation, they refer to the average NFSS for the last 2 ns of the stress-relaxation, and data from

232 three replicates are shown. For unfolding, they refer to the average NFSS at the corresponding

233 unfolding times $t$ (for example, $9.7 \AA$ means averaging across $t=0-2 \mathrm{~ns}$, and $19.7 \AA$ means

234 averaging across $t=9-11 \mathrm{~ns})$.

236 Force curves are recorded from each constant-velocity unfolding replicate using SMD. There

237 thus exist three possible unfolding events which can have varying extent of overlap: (i) concerted

238 breakage of a set of noncovalent interactions, which require a high force/energy; (ii) breakage of

239 noncovalent interactions one by one, or "unzipping", which require a lower unfolding force that

240 spans over a longer range of extension, as well as (iii) breakage of a set of dynamically evolving

241 intramolecular interactions, whose force curve features will likely be diffuse or stochastic and

242 more difficult to interpret. For our RHPs, visual inspection of the unfolding trajectories does not 
243 establish an unambiguous correlation between force curve features - peaks or plateaus - with

244 molecular snapshots. Examination of individual unfolding replicates of a given RHP conformer

245 shows that side-chains have highly dynamic interactions that vary across the five replicates. For

246 example, the solvent-accessible surface area (SASA) evolution over time exhibits different

247 behaviors across the five replicates, even though some force curves exhibit very similar features

248 for a given 20mer RHP (Figure S8). Variable pathways for backbone restructuring are also

249 observed through the evolution of dihedral angles. We can hence suspect that force curve features

250 are a result of a combination of topological variations in the polymer backbone as well as side-

251 chain interactions rather than a one-to-one correlation of a particular force peak to the

252 disassociation of two moieties. Though each RHP sequence conformation replicate has a unique

253 unfolding trajectory, they share commonalities reflected in the averaged force curve from which

254 we can gain insight to the polymer assembly.

255 Since the coil-to-globule transition for hydrophobic homopolymers is well studied, ${ }^{43}$ the force

256 responses for unfolding collapsed homopolymers of chemistries relevant to our RHPs - namely,

257 PMMA and PEHMA - are studied as controls (Figure 4). The unfolding force is rate-dependent in

258 polymeric systems; therefore, the magnitude of the unfolding forces recorded in SMD simulations

259 will be higher than measured by experimental single-molecule techniques which operate at a

260 pulling velocity orders of magnitudes lower. ${ }^{20}$ For PMMA, an initial force peak is present in several

261 of the simulations, and is attributable to the disruption of intrachain dipole-dipole interactions due

262 to PMMA's directional and polar side group. Once the dipole-dipole self-packing interactions are

263 disrupted upon initial unfolding, polar side groups of PMMA can become solvated with water, and

264 it only requires minimal (near-zero) force to unfold the chain further, resulting in a force curve

265 traditionally consistent with PMMA's glassy nature. For PEHMA homopolymers, their force 
266 curves exhibit more features and show a greater variability between the unfolding replicates

267 compared to that of PMMA, demonstrating highly dynamic side-chain interactions, as seen by

268 visual inspection in the simulation trajectories. The averaged force curve for PEHMA Plso $_{50}$

269 displays a characteristic plateau-like behavior at a non-zero force which is ascribed to hydrophobic

270 hydration. ${ }^{9}$

271
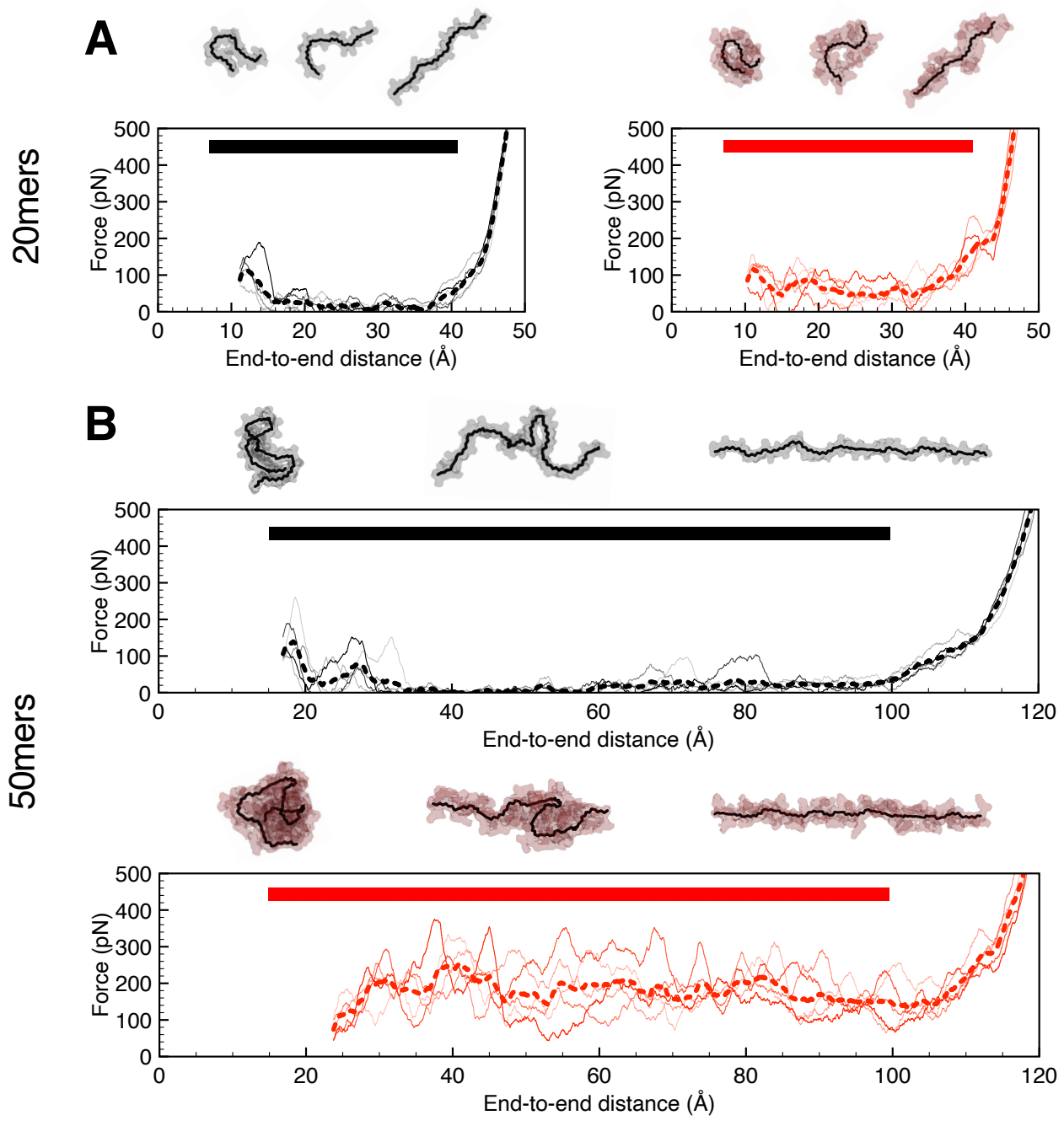

272

273 Figure 4. Unfolding force curves of hydrophobic homopolymers. Force curves for (A) 20mer

274 and (B) 50mer homopolymers PMMA (black) and PEHMA (red) are based on moving averages

275 over 2 - $\AA$ intervals. Shown are the data from five independent unfolding simulation replicates in 
276 different shades of the same color, and the average is given in a thicker dotted line. Example

277 snapshots of initial, intermediate (midpoint), and final conformations of the polymer during

278 unfolding are provided, where side-chains have been rendered semi-translucent to highlight

279 polymer $\mathrm{C}-\mathrm{C}$ backbone topologies.

281 For the multicomponent RHPs, their equilibrated structures are often stabilized by the

282 hydrophobic attraction between EHMA-EHMA side-chains. Some sequences also have 283 conformations stabilized by OEGMA-OEGMA interactions or by a mixture of EHMA and

284 OEGMA interactions, depending on the monomer availability within the chain. Compared to their 285 homopolymer counterparts, 20mer and 50mer RHPs display more varied mechanical responses 286 (Figures 5, S6, and S7). In terms of force curve features, some 20mer RHPs exhibit clear and 287 pronounced force peaks across five replicates, some exhibit a plateau behavior, and some can have 288 more frequent force peaks than others. This demonstrates that the RHP system has an extremely 289 rich energy landscape which can result in vastly different single-chain mechanical responses, even 290 for identical sequences as short as 20 monomers in length. Similarly, force curves of 50mer RHPs 291 also display varying extents of force peaks and plateaus depending on the exact sequence and 292 conformation of the chain. The diverse force curves for unfolding RHPs are distinct from those 293 observed for biological heteropolymers such as proteins or nucleic acids of similar sizes. For 294 example, the unfolding force curve of single-stranded DNA hairpin structures of 55 bases in size 295 reveals a characteristic "rip" feature, indicative of disruption of the hydrogen bonding ${ }^{44}$ This 296 disparity arises from three major design differences between RHPs and biopolymers (e.g. proteins 297 or nucleic acids). First, RHPs do not have intramolecular hydrogen bonding that proves 298 fundamental for the secondary structural formation in proteins. Secondly, RHPs possess several 
299 bulkier and longer side-chains compared to native amino acids that make up proteins (or

300 nucleotides that make up nucleic acids), giving rise to unfolding behavior dominated by side-chain

301 interactions and reconfiguration. Thirdly, RHPs have a racemic mixture of monomers with

302 randomly assigned chiralities - that is, our RHPs are heterochiral - whereas proteins and nucleic

303 acids are intrinsically homochiral. As a result, no characteristic rupture forces from the breakage

304 of specific ordered interactions are observed in RHPs.

305

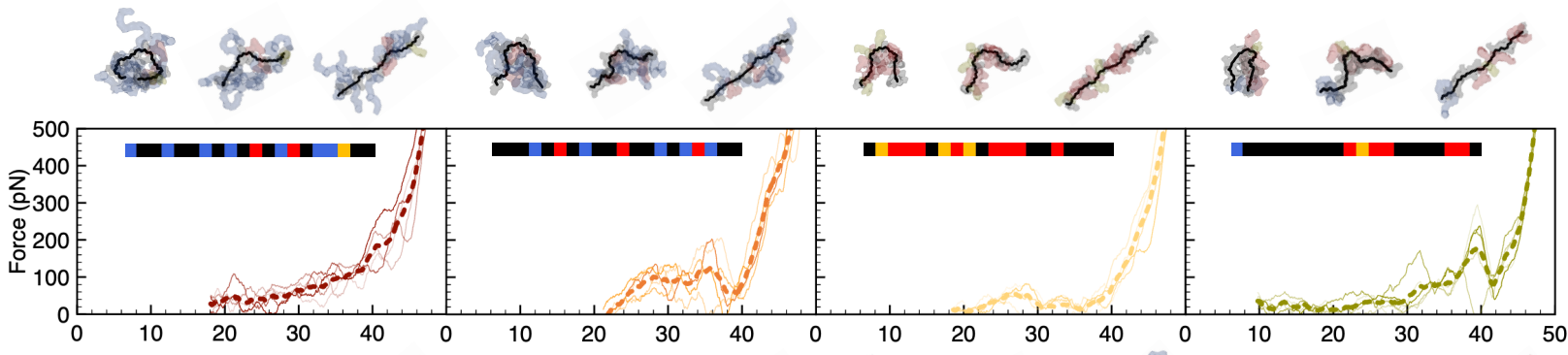

(a)
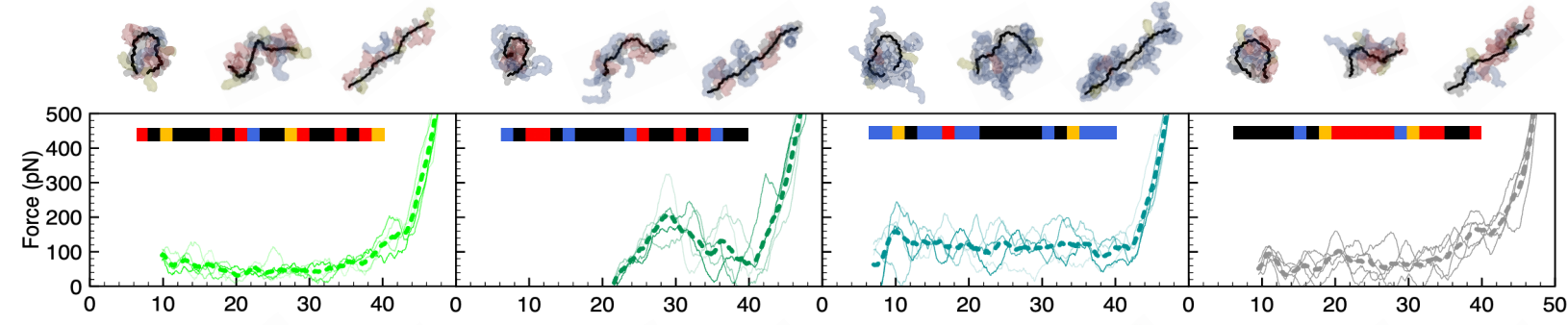

306

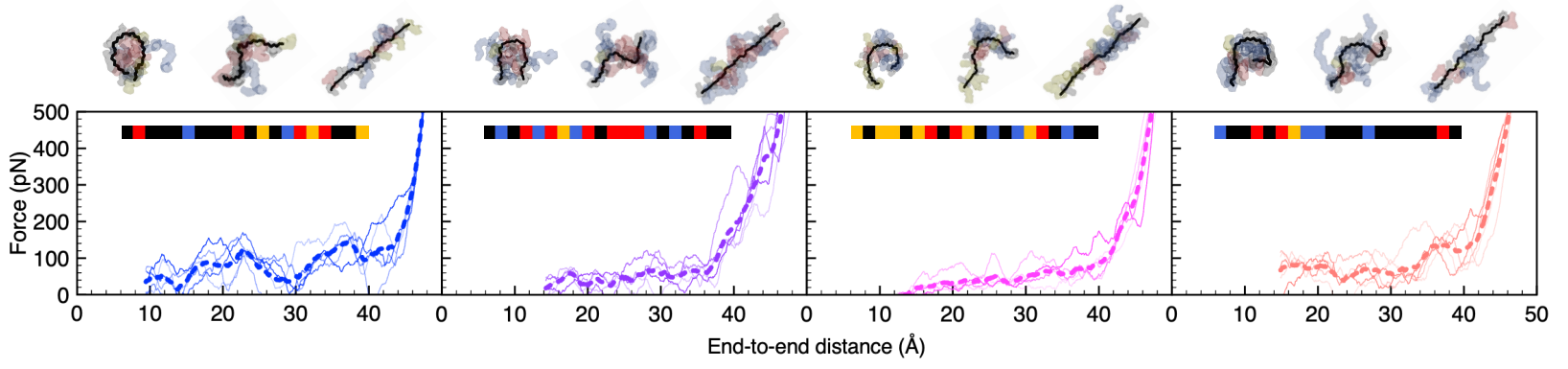

307 Figure 5. RHPs show varied responses to force-induced unfolding. Shown here are the force

308 curves for the unfolding of selected 20mer RHPs with their unfolding trajectory snapshots (initial,

309 intermediate, and final conformations). An exhaustive overview of the unfolding force curves of

310 all 20mer and 50mer RHPs studied are provided in Figures S6 and S7, respectively. 


\section{Monitoring the unfolding pathway and behavior of individual RHP sequences}

313 The unfolding behavior of an RHP is investigated in detail by monitoring the time evolution of

314 dihedral angles and intramolecular contacts to understand backbone restructuring and elucidate the

315 unfolding events. Here, we detail the unfolding pathway of a molten globular 50mer RHP,

316 sequence 12 conformation 8 . As with many 50mer RHPs, the mechanical unfolding of this RHP

317 is mostly driven by the dissociation of the hydrophobic core formed by EHMA-EHMA interactions

318 (Figures 6 and S9). During the first 30-40 ns of unfolding, there are few apparent dihedral angle

319 transitions except for the two ends. In fact, the internal structure of the RHP is well preserved

320 during the initial mechanical perturbance, confirmed by the preservation of contacts from pulling

321 time $t=1 \mathrm{~ns}$ to $30 \mathrm{~ns}$ (Figure 6C). Therefore, conformational changes are possibly mediated by

322 the extension of the two chain ends which possess higher mobility as demonstrated by unbiased

323 MD simulations and by diffuse, small changes throughout the entire chain in order to enable the

324 initial increase in end-to-end distance. On a morphological level, the globule becomes somewhat

325 distorted under the applied force. From $t=50$ to $60 \mathrm{~ns}$, the chain undergoes segmental separation

326 where the EHMA hydrophobic core dissociates into two separate, smaller EHMA-stabilized cores.

327 Examining the dihedral evolution, this is believed to be mediated by the extension of the MMA

328 block (highlighted by red boxes in Figure 6C), as indicated by the distinct gauche to trans angle

329 transition. This pearl-like intermediate is similar in shape to that predicted by theory ${ }^{13,14}$; however,

330 in the case of this specific RHP, a hydrophobic MMA block instead of a hydrophilic cluster unfolds

331 first. Upon segmental separation, the extensions of the two smaller units unfold sequentially, where

332 the longer EHMA segment near the beginning of the sequence has persistent hydrophobic

333 interactions until $t=90 \mathrm{~ns}$ and unfolds last (highlighted by green boxes in Figure 6C). Overall, 
334 during the latter stages of the unfolding pathway (after $t=50 \mathrm{~ns}$ ), EHMA side-chains, which

335 previously had longer range hydrophobic interactions, have an increased number of local

336 interactions due to their physical proximity within the sequence, giving rise to the emergence of

337 some gauche angles in the more extended conformation. Analyses on the four other replicates of

338 this RHP sequence and conformation also show relatively similar unfolding pathways; however,

339 the segmental separation can take place at a different MMA block via its extension (Figure S10).

340 This suggests that the MMA blocks can behave as a hinge, such as the $\alpha$-helical linker in $\operatorname{spectrin}^{5}$,

341 to mediate or propagate unfolding. This is supported by our finding that PMMA homopolymers

342 require minimal force to unfold, leading MMA blocks within an RHP to be more amenable to

343 reconfigure during unfolding. Nevertheless, it should be noted that MMA blocks do not always

344 behave in this manner in the sequences investigated, and there are a myriad of complex interactions

345 that would allow alternative unfolding pathways to exist, making this phenomenon highly

346 sequence- and conformation-specific.

A

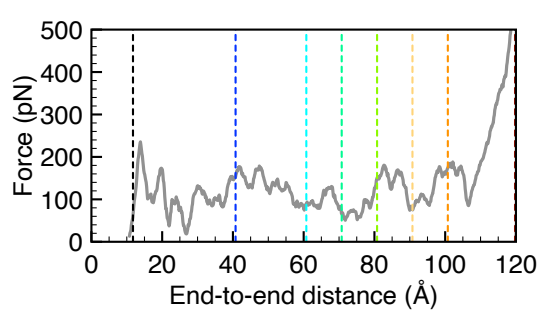

B

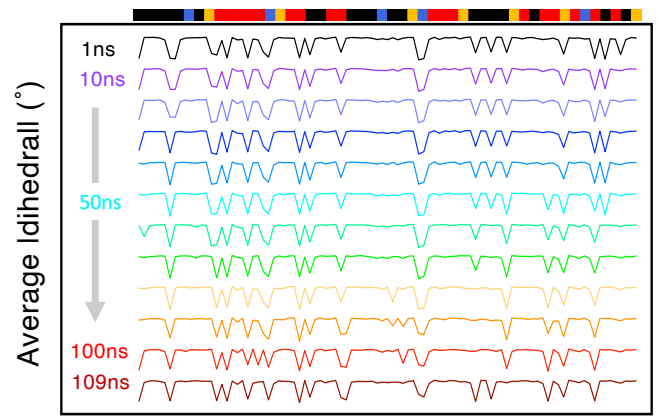

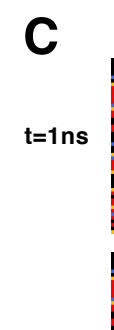

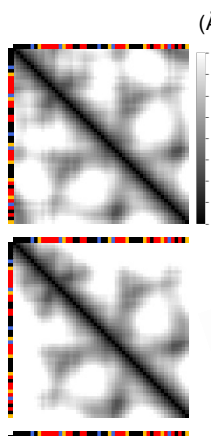

(A)
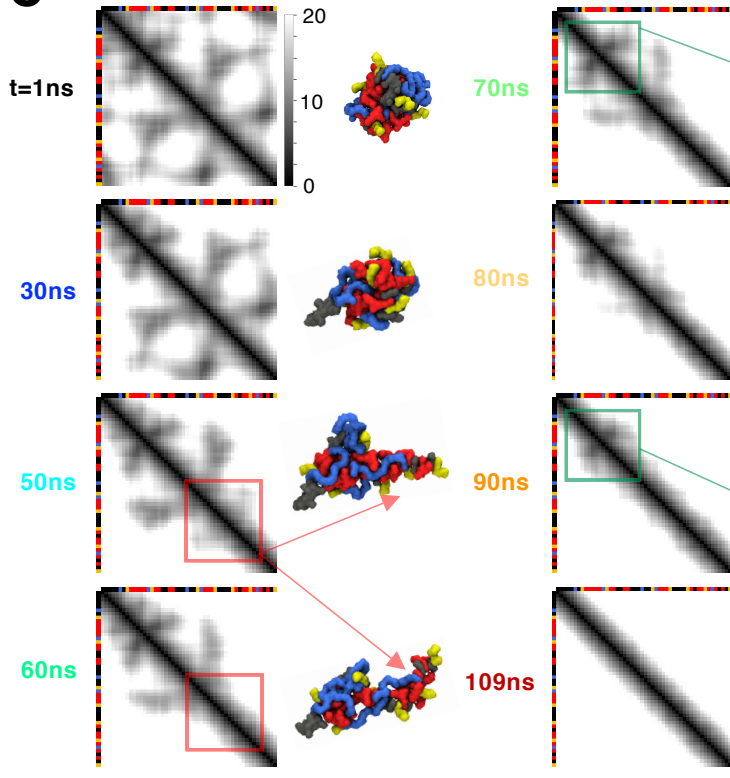

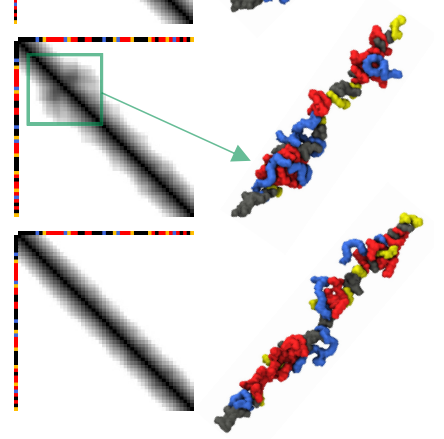


349 Figure 6. Monitoring the unfolding pathway of a 50mer RHP. This particular RHP corresponds

350 to sequence 12 conformation 8 , and results from a representative unfolding replicate is shown here.

351 (A) Unfolding force curve. Vertical dotted lines indicate specific time points of interest. (B)

352 Backbone dihedral angle evolution for the entire unfolding process of this RHP. (C) Selected 2-

353 ns-time-averaged $C_{\alpha}$-based contact maps. Red/green boxes highlight regions of interest.

354 Corresponding VMD snapshots of the RHP are also shown to visualize the unfolding events. The

355 unfolding trajectory showing the unfolding intermediates per every $10 \mathrm{~ns}$ is provided in Figure S9.

356 The unfolding trajectory of this RHP visualized in VMD is provided in Supporting Information

357 Movie S1.

359 Another finding observed in the study of sequence 12 conformation 8, as well as several other 360 compositions and configurations, is that upon RHP unfolding, OEGMA side-chains wrap around

361 the surface of the main RHP chain. Throughout the unfolding trajectory, different OEGMA side-

362 chains preferentially interact with hydrophobic regions, as highlighted in Figure 7, where darker

363 regions in the contact maps calculated from side-chain centers of mass denote the physical

364 proximity between the side groups of each monomer. Since the contact maps are time-averaged

365 over $2 \mathrm{~ns}$, the residues have significant residence times rather than being coincidental

366 instantaneous occurrences. OEGMA, shown to be amphiphilic in nature ${ }^{15,45}$ and whose side-chains

367 possess high conformational flexibility, can establish a favorable interface between the

368 hydrophobic monomers and the water molecules, serving as a possible protection mechanism via

369 solvent shielding. To obtain direct, quantitative evidence of OEGMA wrapping, separation

370 distances between the tail atoms of OEGMA and EHMA residues are computed and plotted against

371 time for all possible OEGMA/EHMA pairs in the RHP sequence (Figure 7C). Small separation 
372 distances between the two tail atoms can arise either from sequence effects due to mere geometric

373 proximity between covalently bound monomers within the chain or from the wrapping phenomena

374 we intend to capture. To decouple the two effects, EHMA monomers have been grouped into four

375 groups based on their positions in this particular sequence. The fourth OEGMA monomer,

376 highlighted in magenta, is actively involved in the wrapping process, as observed from the

377 nonmonotonic time-dependence in the separation-distance curves with multiple regions of EHMA

378 (Figure 7). OEGMA-EHMA distance curves for the other OEGMA residues do not manifest the

379 same extent of wrapping (Figure S11). Moreover, this wrapping phenomenon has been observed

380 for numerous 20mer and 50mer RHPs studied, establishing its relative generality. Whilst this

381 observation is novel for our particular chemistry, the solvent-shielding phenomenon is reminiscent

382 of previously studied interactions between PEG - which makes up the long OEGMA side-chain

383 - and biomolecules. Conjugation of proteins with PEG chains (PEGylation) and similar

384 molecular brushes is a method to enhance the thermal and mechanical stability of certain proteins

385 using covalent modifications. ${ }^{46-48}$ While comprehensive studies providing mechanistic insights are

386 limited, DeBenedictis et al. report that PEG chains can wrap around an $\alpha$-helical protein and shield

387 water molecules from attacking the hydrogen bonds, delaying the unfolding process.$^{49}$ Moreover,

388 they show that the PEG chains disproportionately favor hydrophobic and charged residues, 389 phenomenologically similar to the OEGMA wrapping in our system. 


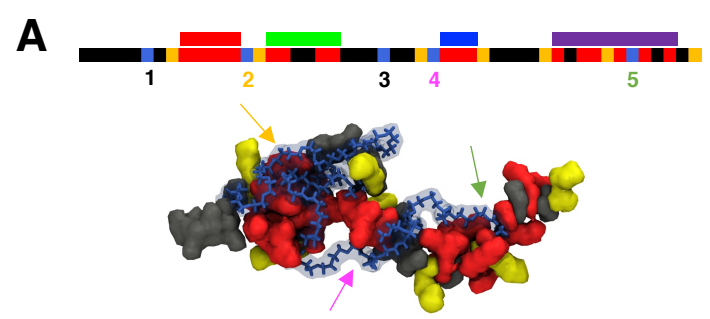

B

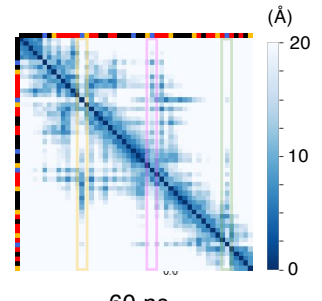

$60 \mathrm{~ns}$

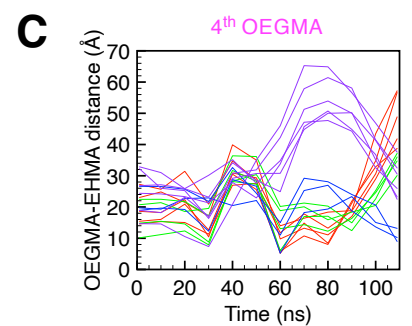

392 Figure 7. OEGMA side-chain wrapping is observed during RHP unfolding. (A) RHP

393 sequence schematic (sequence 12) and a structural snapshot at pulling time $t=50$ ns. EHMA

394 monomers within this sequence can be approximately grouped into four sets, color-coded in red,

395 lime green, blue, and purple. OEGMA monomers are numbered and color-coded as shown in the

396 sequence. The same color-coding is used to indicate OEGMA monomers in the snapshot, showing

397 OEGMA wrapping around hydrophobic EHMA and charged SPMA residues. The OEGMA side-

398 chains have been rendered semi-translucent and water has been excluded for ease of visualization.

399 (B) A time-averaged side-chain-based contact map showing preferential interactions of some 400 OEGMA side-chains with hydrophobic residues. (C) Time evolution of tail-atom separation 401 distances for the second OEGMA monomer to the other EHMA monomers based on 2-ns-time402 averaged tail-atom contact map data. Therefore, each line in the plot corresponds to the tail-atom 403 distance of one particular OEGMA/EHMA pair. Similar plots for the other OEGMA monomers 404 can be found in Figure S11. 
Differently positioned OEGMA monomers have side-chains wrap around the main chain to

407 varying extents at different time points of unfolding. Consequently, the wrapping behavior is not

408 a one-size-fits-all process, and sequence effects play an important role to enable the protection

409 mechanism at a given time point. This is analogous to protein PEGylation, where the site of

410 conjugation in a protein also affects the extent of mechanical reinforcement imparted by PEG

411 chains.$^{50,51}$ For our RHPs, per the side-chain-based contact maps provided in Figure S11, it may be

412 hypothesized that OEGMA monomers surrounded by MMA monomers are less prone to partake

413 in the proposed protection mechanism via side-chain wrapping. Previous analysis has shown that

414 OEGMA monomers in equilibrated RHPs are better solvated when they are surrounded by MMA

415 residues compared to when surrounded by EHMA residues ${ }^{15}$, corroborating our observations.

416 Since EHMA is more hydrophobic than MMA, side-chain wrapping around the EHMA residues

417 to minimize water contact is energetically beneficial for the system, suggesting a protection

418 mechanism at play.

419 Physicochemical factors affecting RHP unfolding

420 A variety of physicochemical parameters, including chain length, chemical composition, 421 sequence characteristics, and backbone topology, can influence the RHP unfolding pathway, force 422 curve features, mechanostability, and nonequilibrium unfolding work. Specifically, RHP 423 mechanostability, or the mechanical resistance of RHPs to the applied tensile force, is 424 characterized using peak forces and their distributions. First, on average, RHPs of 50 monomers

425 in length are found to be more mechanically stable than the 20mers (Figure S12). This can be 426 reasoned from the previous conclusion that 50mers typically begin from a state of greater chain 427 compactification due to lower solubility and higher propensity for hydrophobic collapse. As a 428 result of such compactification, the unfolding pathways of 20mers and 50mers also differ. Many 
429 20mer RHPs and homopolymers can unfold via an $\Omega$-shaped topological intermediate (Figure 5).

430 Since this is common to sequences having different chemical compositions and sequence traits,

431 the $\Omega$-shaped intermediate may have a topological origin given that most compact 20mers

432 investigated initially assume a U- or O-shape, where the 2D projection of an RHP backbone onto

433 any surface gives a U-shape if there is no intersection and an O-shape if there is one intersection.

434 Notably, an unfolding pathway phenomenologically similar to the pathway proposed here has been

435 observed during the mechanical unfolding of biological $\beta$-hairpin structures ${ }^{52,53}$, despite the fact

436 that specific hydrogen bonding is responsible for stabilizing $\beta$-hairpins whereas nonspecific

437 hydrophobic interactions are responsible for stabilizing our RHPs. 50mer RHPs, on the other hand,

438 have more diverse topologies and, as a result, more diverse unfolding behaviors. Previous

439 theoretical analysis on amphiphilic heteropolymer unfolding suggests the existence of a pearl-

440 necklace unfolding intermediate due to favorable solvation in hydrophilic residues and unfavorable

441 solvation in hydrophobic ones. ${ }^{13,14}$ However, this is rarely seen in the multicomponent RHPs. When

442 the pearl-necklace unfolded intermediate is observed, we find that the pearl formation is not

443 necessarily due to the unfolding of hydrophilic cluster(s) within the polymer sequence; rather,

444 reduced steric hindrances in MMA segments facilitate unfolding through a linker-like mechanism

445 as discussed in the case study. In addition, we suspect that the solvent-shielding protection

446 mechanism discussed previously can alter the unfolding pathway by modulating the barriers

447 pertinent to the water solvation energetics, thus eliminating the need for a necklace structure during

448 the force-induced globule-coil transition. ${ }^{54}$ Therefore, we find that, in addition to hydrophobicity

449 and hydrophilicity of the monomers, their exact chemistry and sterics impact the RHP unfolding

450 response, which would not have been captured by coarse-grained simulations and theories. 
Our RHPs possess high chemical heterogeneity with lengthy and/or bulky side-chains that prove

452 impactful to their unfolding responses. Since chemical composition, sequence characteristics, and

453 backbone topologies cannot be easily decoupled for our statistically random polymers, these are

454 examined holistically to provide insights into their effects on unfolding and mechanostability. The

455 effect of chemical composition is investigated by examining the monomer content in each chain

456 using pooled data across sequences (Figures S13 and S14). No strong correlations are observed

457 for 20 or 50 mer sequences, indicating that chain composition alone cannot dictate the mechanical

458 response of a chemically heterogeneous RHP. One might expect that, as the most prevalent

459 stabilizing interactions in RHPs are EHMA-EHMA hydrophobic attraction, its content would have

460 a high correlation with mechanostability. However, even though PEHMA homopolymer is

461 relatively mechanically stable, having a high fractional content of EHMA in the RHP does not

462 guarantee the same. A weak negative correlation can be observed between SPMA content and

463 RHP mechanostability due to electrostatic repulsions, though RHPs with the same chemical

464 composition (20mer RHP sequences 9 and 13) can produce disparate mechanical responses. These

465 results hint that sequence and/or topological effects may be of more relevance than chemical

466 composition for mechanostability.

467 The work required for unfolding an RHP is calculated as the area under the force curve, with the

468 integral evaluated from initial end-to-end distance to $45 \AA$ for 20 mers and $110 \AA$ for 50 mers.

469 Unfolding work is rate-dependent for polymeric systems, but can nevertheless provide information

470 on the internal friction in polymeric globules, which in turn reflects the roughness of their

471 conformational energy landscape.$^{8,55,56}$ Figure 8 maps the specific unfolding work against the

472 change in specific total SASA upon polymer unfolding for homopolymers and RHPs, where

473 "specific" properties are normalized by the polymer molecular weights. The change in SASA 
474 should encompass effects of both molecular weight and overall hydrophilicity, allowing each

475 sequence to be compared. We notice that there are several cases where $\triangle$ SASA/MW is negative

476 (Figure 8), all of which come from different RHP unfolding replicates. This can be explained by

477 the amphiphilic nature of OEGMA side-chains as well as the fact that OEGMA is a main

478 determinant of the magnitude of the overall SASA as it has lengthy side-groups. The PEG chains

479 in OEGMA residues can either wrap around the main chain as previously discussed, giving rise to

480 relatively low values of total SASA values, or become fully solvated, giving rise to high SASA.

481 The balance between the two at the start and end of the unfolding trajectory then partly dictates

482 the magnitude of $\triangle \mathrm{SASA}$ - where some of which can be negative in value. First, comparing 20mers

483 and 50mers, the longer polymers which compactify more require a greater specific unfolding work.

484 In addition, relative to the limits established by the homopolymers, namely PMMA and PEHMA

485 specific unfolding work, the 50mer data also has a tighter distribution. Our chemically

486 heterogeneous 50mer RHPs experience relatively uniform compactification and are more dense

487 compared to 20mers, suggesting that the specific unfolding work correlates well with polymer

488 compactness and molecular weight. This has also been demonstrated on unfolded or disordered

489 proteins that the magnitude of internal friction correlates with protein compactness. ${ }^{55}$ Closer

490 examination of the nonequilibrium unfolding work shows that there are minimal trends relating

491 unfolding work to the chemical composition of the polymer chains and corroborate the observation

492 that all of the 50mer RHPs studied have similar extents of internal friction (Figure S15). A weak

493 positive correlation exists for unfolding work and the number of OEGMA monomers in a 20mer

494 RHP. Since OEGMA has long side-chains, giving a brush-like architecture, it will have the greatest

495 contribution to internal friction which in our case would predominantly be due to intramolecular 496 side-chain interactions. 


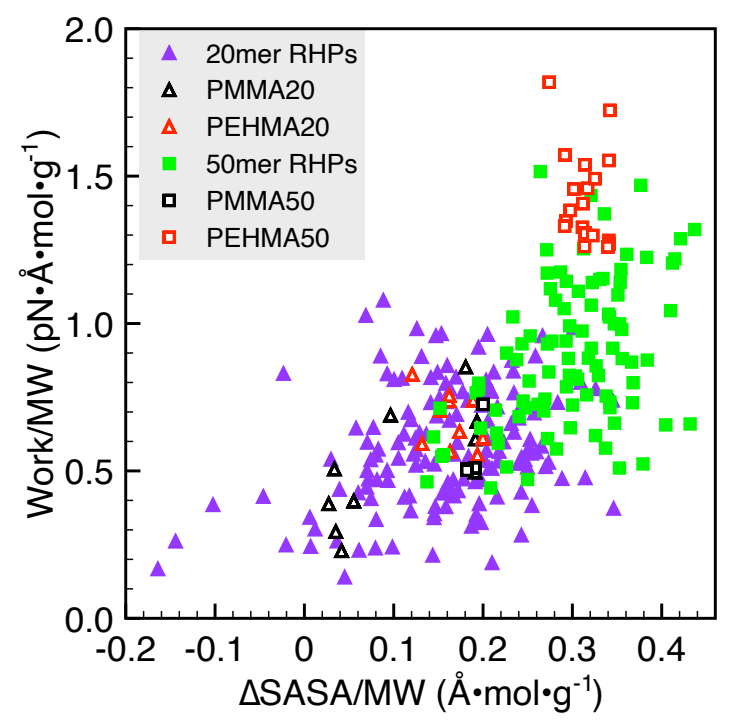

499 Figure 8. Specific non-equilibrium unfolding work (work / molecular weight MW) for 500 homopolymers and RHPs versus the change in specific total SASA (DSASA / MW). $\triangle$ SASA

501 is calculated by subtracting the average total SASA of the polymer in the first 2 ns of the unfolding

502 simulation from that in the last 2 ns of the simulation. Individual data points are from each 503 unfolding replicate, including five replicates for each sequence of thirty 20mer RHP 504 sequence/conformation, nineteen 50mer RHP sequence/conformation, two PMMA 20 , one $505 \mathrm{PMMA}_{50}$, two PEHMA 2 , and four PEHMA $\mathrm{PH}_{50}$ A similar plot where calculated unfolding work is 506 based on a defined chain extension interval is shown in Figure S16.

508 Examining the effect of sequence characteristics is less obvious for our data, yet postulates can 509 be put forward. Chains with alternating hydrophilic (OEGMA or SPMA) and hydrophobic (MMA 510 or EHMA) monomers were likely to display force curves that are generally increasing in a 511 monotonic fashion without pronounced features (Figure 4). Similarly, sequence effects on the 512 mechanical response of 50mer RHPs can be rationalized on a case-by-case basis, albeit not 
513 deterministically. RHPs with high SPMA content and SPMA spaced out within a chain result in

514 very mechanically labile unfolding because electrostatic repulsions between anionic groups

515 facilitate the hydration and unfolding of the chain. Yet, there are exceptions, and this is not the

516 case for sequence 5. The destabilizing effect of having a relatively high SPMA content is

517 counteracted by high hydrophobic content, leading to more compact chain morphology upon

518 hydrophobic collapse and giving rise to pronounced peak features. To enable a predictable power

519 for rational design of the multicomponent RHPs, further and more systematic analysis on sequence

520 characteristics is needed. This points to future work on developing statistical models that can be

521 used to assess and quantify sequence traits. Similar analysis has been carried out in protein

522 homology considering the vast number of possible permutations of the amino acid constituents,

523 which can be informative when investigating the RHP systems. ${ }^{57}$

524 Topological design in heteropolymer systems is an emerging topic in macromolecular

525 engineering. ${ }^{58,59}$ Our RHPs provide a library of sequences with distinct metastable conformational

526 states stabilized by reversible intramolecular interactions, offering opportunities to decouple

527 sequence and topological effects. We first select two compact 20mer conformers and compare

528 their peak force distributions (Figure S17), noting that different initial equilibrated structures of

529 identical 20mer sequences can have dissimilar mechanical responses to the tensile stimulus. While

530 20mers have backbones that are topologically simple and similar, 50mers have more diverse

531 conformations and topological organizations and can further shed light on how polymer topology

532 affects the single-chain mechanics of RHPs. Four conformations each of the homopolymer

533 PEHMA and of two RHP sequences (sequence 15 and sequence 19) have been investigated. Visual

534 inspection of the different initial conformations reveals that the homopolymers are more spherical

535 in shape - though the exact chain topologies differ for the four conformations, whereas the two 
536 heteropolymers in question adopt more varied morphologies (Figure 9). For both homo- and

537 heteropolymers, unique chain topologies result in distinct mechanical responses to forced

538 unfolding (Figure 9). This aligns with previous results on proteins, whose native topology can

539 greatly impact its unfolding behavior, as the initial chain topology restrains the progression of

540 unfolding events that can take place. ${ }^{60-62}$ For the heteropolymers, the effect of chain topology is

541 more evident. Structure 2 of sequence 15 has a slightly planar morphology, and the two chain ends

542 point in opposite directions. As a result, mechanical unfolding of this conformation leads to an

543 initial resistance, followed by a drop to near-zero force, indicating nearly spontaneous unfolding

544 behavior at relatively high chain extension. Closer scrutiny of the initial resistance reveals two

545 contributing factors. During initial mechanical perturbance of the polymer, the planar-like

546 morphology deforms laterally and becomes more prolate/ellipsoidal (Figure S18). The internal

547 friction from OEGMA side-chain interactions likely contribute to the initial peak observed in the

548 averaged force curve. A tadpole-like unfolding intermediate, where a globule-shaped head is in

549 coexistence with a stretched chain end ${ }^{63}$, is observed at around $t=30 \mathrm{~ns}$ for this structure but not

550 the other topologies of the same RHP sequence. At around $t=40-45 \mathrm{~ns}$, EHMA-EHMA

551 hydrophobic interactions become disrupted to allow further chain extension, after which force

552 drops to a near-zero value as essentially all stabilizing intramolecular interactions have been

553 disrupted, and no further pronounced force peaks are observed (Figures 9B and S18). On the other

554 hand, prolate structures of the heteropolymer (structures 2 and 3) can display a relatively sustained

555 mechanical resistance over a large range of end-to-end distances (Figure 9). Sequence 19, which

556 is high in negatively-charged SPMA content, is chosen as another RHP for investigating the effect

557 of topology. While two of the native conformations (structures 1 and 3 ) unfold easily, mostly

558 attributable to electrostatic repulsion between anions which facilitates unfolding, the remaining 
559 two conformations display pronounced force peak features in their unfolding force curves.

560 Therefore, even with high SPMA content, there may exist topologies for which high

561 mechanostability may be achieved. Interestingly, structure 4 of sequence 19 also requires the

562 highest force ( $285 \mathrm{pN}$ on average, with the highest being $410 \mathrm{pN}$ in one of the replicates) to unfold

563 compared to the different conformers of PEHMA or sequence 15. We hypothesize that, for this

564 topological organization of the backbone, the arrangement of negatively-charged sulfate ions

565 within the SPMA side-chains amplifies the strength of the hydrophobic attraction, similar to

566 previous experimental and simulation results proving the modulation of hydrophobic effect by

567 proximal (i.e., within $1 \mathrm{~nm}$ ) covalently-attached charged moieties in an ion-specific fashion. ${ }^{64,65}$

568 For this RHP conformation (sequence 19 structure 4), its unfolding requires the cooperative

569 breaking of multiple types of interactions at approximately the same time, leading to one

570 pronounced force peak at low extension (Figure 9C).

571 Comparing the intramolecular contact evolutions of the unique topological conformations

572 (Figures 9 and S19) offers additional insight. First, both the time-evolution of contact maps and

573 the contact reduction trends are similar between replicates of a specific topology for a given

574 polymer sequence. For the RHPs, this would suggest that, once we have a metastable structure,

575 there exists a generally consistent energetic pathway for unfolding, though small deviations can

576 exist. Secondly, we find that homopolymer unfolding leads to both dissolution and reformation of

577 contacts whereas heteropolymer unfolding is predominantly mediated by the dissolution of contact

578 features. Thus, backbone restructuring is more common in the homopolymer compared to in the

579 heteropolymer upon unfolding, following the mobility trends obtained in previous unbiased

580 simulations. ${ }^{15}$ From an enthalpic point of view, monomer-monomer interactions are all identical

581 within homopolymers, whereas heteropolymers have a multitude of possible interactions between 
582 the different monomer types. These inter-monomeric potentials lead to a much more textured

583 energy landscape of the evolving RHP conformations since there are now ten unique monomer

584 pairings rather than the single homopolymer self-interaction. Polarity, electrostatics, and

585 hydrophilicity lead EHMA-EHMA and OEGMA-OEGMA associations to be favored while

586 SPMA-SPMA and SPMA-EHMA interactions are avoided. From a topological perspective, all

587 four conformations of PEHMA investigated possess an initial antiparallel topology (contacts

588 forming a line perpendicular to the main diagonal), which then induces an unzipping-type behavior

589 by reconfiguring to a helical-like topology (contacts residing next and parallel to the main

590 diagonal). This unfolding response via unzipping is enabled by the high entropic conformational

591 flexibility of the backbone as well as that of the side-chains evidenced by the gradual reduction in

592 intramolecular contacts for homopolymers, while the heteropolymers show more varied contact

593 reduction rates throughout the trajectories (Figure 9). Moreover, force peak occurrences appear to

594 correlate with a rapid reduction in intramolecular contacts, with the magnitude of the force peak

595 being dependent on the nature of monomer-monomer interactions disrupted upon unfolding. There

596 is also greater variability in the contact reduction curve behavior between each topological

597 conformation as well as between replicates of the same conformation for the RHPs compared to

598 the PEHMA homopolymer.

599 
$1 \quad 2 \quad 3 \quad 4$
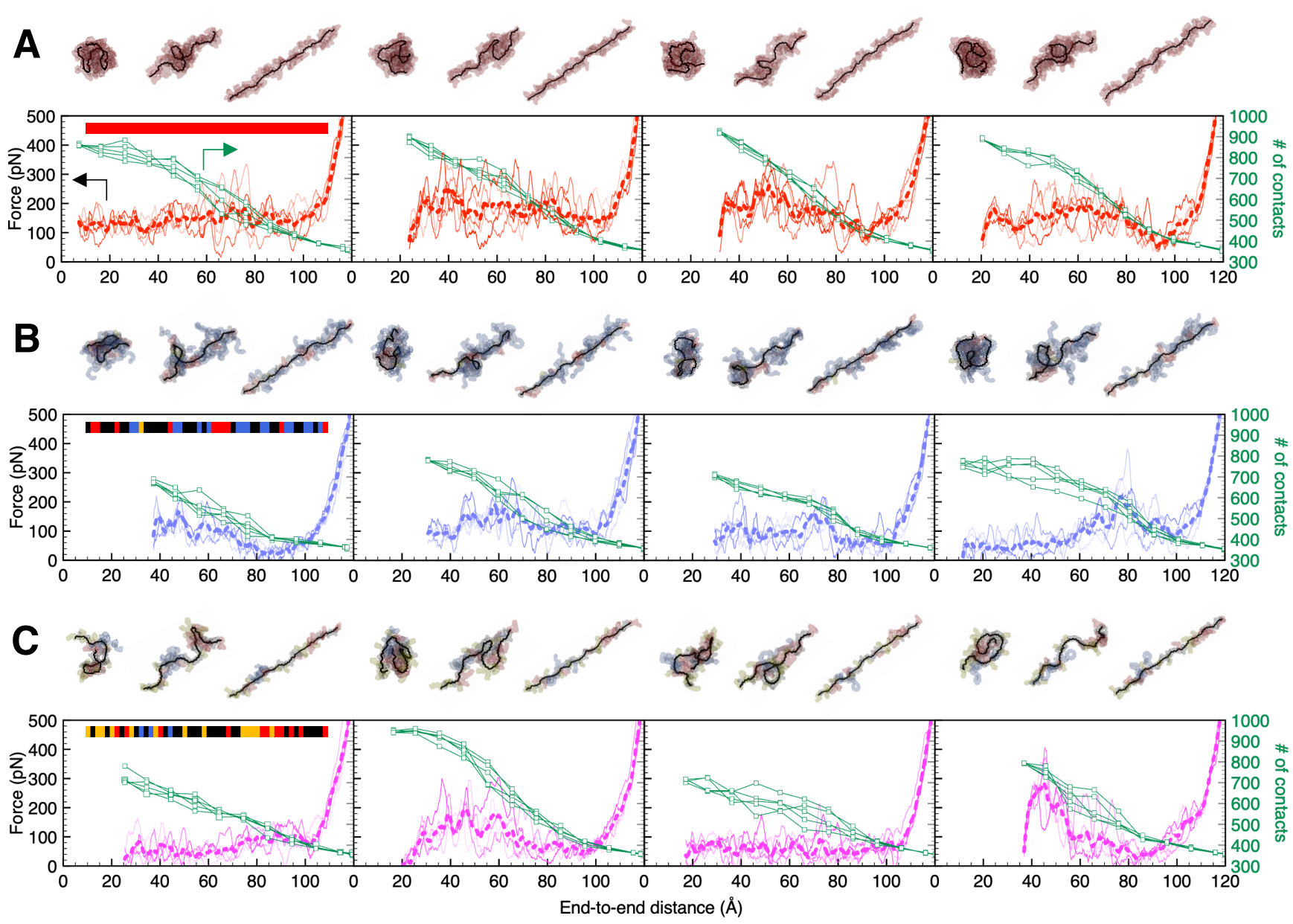

601 Figure 9. Backbone topology affects RHP unfolding responses. Unfolding force curves of four

602 different starting conformations, numbered 1-4, of (A) homopolymer PEHMA and (B, C) RHP

603 sequence 15 and 19. Superimposed are contact data (in dark green) showing the reduction in the

604 number of intramolecular contacts during RHP unfolding (one curve for each replicate; see

605 Methods for details). Corresponding contact map evolutions and contact reduction curves in a

606 different presentation style can be found in Figure S19.

608 For further comparison of chemical and topological contributions to unfolding behavior, we

609 compare the dihedral angle dynamics between the different polymer sequences and between 
610 conformations. The standard deviation of Idihedral anglesl across the entire unfolding trajectory

611 can be used as a proxy for the extent of backbone reconfiguration, allowing identification of

612 mechanically stable regions (i.e., chain segments that undergo minimal transition in all five

613 unfolding replicates) within a polymer chain (Figure S20A-C). Both PEHMA and RHP sequences

614 showed regions that remained mechanically stable from each initial topology. However, in the

615 RHPs, the segments closest to the chain ends offered a disproportionately consistent opportunity

616 for reconfiguration. Further analysis of the chiral nature of the monomers along the sequence

617 revealed that the emergence of mechanically stable segments correlates with alternating chirality

618 in the residues (Figure S20D). Additionally, both intra- and inter-monomeric dihedrals can mediate

619 conformational changes upon mechanical unfolding with no preference of one over the other

620 (Figure S21). This highlights the capability of atomistic MD simulations to capture the high

621 conformational flexibility of the polymer backbone. Overall, our results demonstrate that, for a

622 multicomponent polymeric system with topological heterogeneity, both the backbone

623 conformation and sequence are important factors in affecting the single-chain unfolding response,

624 with topological effects capable of outweighing chemistry or sequence effects.

625 The above analysis highlights the importance of topology in affecting the unfolding response,

626 pathway, and dynamics of single-chain heteropolymer systems. Additionally, topological design

627 can be a viable option to tune the mechanical response of a heteropolymer. In practice, both internal

628 and external confinement strategies can be employed to force single-chain heteropolymers into

629 certain topologies ${ }^{59}$ Internal confinement utilizes intramolecular crosslinking and/or orthogonal

630 chemistry. Perez-Baena et al. have used long bifunctional crosslinkers via thiol-yne coupling

631 reaction to promote the formation of long-range loops in compactified SCNPs. ${ }^{66}$ External

632 confinement refers to physical confinement of the polymers at the nanoscale, consequently altering 
633 the configurational sampling space of the system..$^{39,59}$ Therefore, synthesis of high concentrations

634 of RHPs within a nanofluidic device with optimized channel geometries may be of interest in

635 future experimental work for directed topological RHP synthesis. Processing conditions can also

636 be coupled to the aforementioned strategies to assist formation of desired topological structures. ${ }^{67}$

637 To gain further understanding of how RHP topological motifs might correlate with force curve

638 features, emerging concepts such as circuit topology and graph theory could be applied ${ }^{58}$.

639 Additionally, a greater variety of RHP sequences with various chemistries and characteristics

640 could provide enough data for a rigorous quantitative model, though computational costs of the

641 pulling simulations remain a hurdle. As demonstrated in one RHP (sequence 19), the realization

642 that spatially-defined anions within a fold can modulate the strength of hydrophobic attraction

643 should also promote new research into this area by combining topological control with sequence-

644 defined polymer synthesis. This would allow us to design in structural (in)stabilities for relevant

645 applications for synthetic heteropolymers or SCNPs self-assembled through hydrophobic collapse.

\section{CONCLUSIONS}

647 In summary, this work investigates the structure and single-molecule mechanics of protein648 inspired RHPs in water using all-atom simulations. The four-component heterochiral RHPs sample

649 from a broad statistical distribution of metastable conformations, and so do their properties. As a

650 result, their structure-property landscape proves highly complex, and there is no singly defined

651 response to forced unfolding for the RHPs. Nevertheless, our data suggest that the physicochemical

652 parameters of the RHPs, particularly the backbone topology, can be tuned to enable specific

653 unfolding responses, which may be leveraged to mediate specific interactions with

654 biomacromolecules. As the RHP system presented here also belongs to the active field of SCNPs, 655 our findings on the chemical heterogeneity in polymer design, topological organization of the 
656 polymer backbone, and the importance of side chain length and bulkiness in modulating polymer

657 behavior may open doors for further research in this area. More generally, this work highlights the

658 necessity of atomistic details in elucidating single-molecule mechanics of multicomponent

659 heteropolymers, revealing phenomena (such as the existence of a dynamic multitude of unfolding

660 pathways as well as the OEGMA wrapping as a protection mechanism) that cannot be easily

661 captured by previously proposed theories. Overall, heteropolymer systems with high chemical and

662 conformational heterogeneity, such as the one presented here, necessitate further exploration.

\section{METHODS}

\section{Unbiased molecular dynamics (MD) simulation details}

665 RHP sequences with degrees of polymerizations of 20 and 50 (referred as 20mers and 50mers, 666 respectively, in this work) were simulated by selecting the first 20 and the first 50 residues of 667 100mer RHPs with target compositions of MMA:OEGMA:EHMA:SPMA in ratios of either $66850: 25: 20: 5$ or 50:5:30:15 by number generated and parameterized per methods in Hilburg et al. ${ }^{15}$ 669 Annealing protocol initially minimized and equilibrated at $500 \mathrm{~K}$ for $40 \mathrm{~ns}$ and then ramped down 670 to $300 \mathrm{~K}$ over $40 \mathrm{~ns}$ in implicit solvent. This was repeated ten times and each resulting structure at $671300 \mathrm{~K}$ was extracted for explicit solvation in a periodic octahedral geometry with approximately 67240,000 molecules of SPC/E water and potassium counterions (to offset SPMA charges). Each 673 structure was then annealed to $650 \mathrm{~K}$, held for $20 \mathrm{~ns}$, and cooled down to $300 \mathrm{~K}$ over $40 \mathrm{~ns}$. The 674 structures were then held at $300 \mathrm{~K}$ for $60 \mathrm{~ns}$, the latter $40 \mathrm{~ns}$ of which were used for analysis. The

675 final frames of these trajectories were then used for unfolding simulations.

676 Steered molecular dynamics (SMD) simulations

677 SMD simulations were performed on the obtained equilibrated structures using a constant678 velocity protocol in order to mechanically unfold the RHPs in explicit water at $300 \mathrm{~K}$. For all RHP 
679 sequences and conformations studied, the two ends are defined to be the backbone $C_{\beta}$ atom of the

680 first monomeric unit and the backbone $C_{\alpha}$ atom of the terminal unit (Figure S2). A biasing potential

681 is applied to the two ends with a force constant of $7.0 \mathrm{kcal} \mathrm{mol}^{-1} \AA^{-2}$, pulling the two ends apart at

682 a constant speed of $1.0 \AA \mathrm{ns}^{-1}$. Other parameterizations, including Langevin thermostat and

683 Berendsen barostat, remain identical to those reported in Hilburg et al. ${ }^{15}$ All 20 mer RHPs are

684 stretched until the end-to-end distance reaches $50 \AA$, and all 50mers are stretched until $120 \AA$,

685 where there are no apparent interactions between non-adjacent monomers. Five independent

686 replicates initiated with new random velocities were performed for all RHP sequences studied.

687 The choices of simulation parameters have been informed by previous works involving the use of

688 SMD (Table S1).

689 In silico stress-relaxation experiments were performed to gain insights into the dissipation of

690 induced tensile stress from the unfolding process of single-chain RHPs. Snapshots of interest were

691 selected at particular time points from throughout the one-stage pulling trajectories and used to

692 obtain the atomic coordinates. Randomized velocities are used to initiate the stress-relaxation

693 simulations. The restraint on the end-to-end distance in SMD is set to be a constant, equivalent to

694 that at which the snapshot was extracted, and RHPs are allowed to relax at that restrained end-to-

695 end distance for 20 ns. For each snapshot, three independent stress-relaxation experiments are

696 reported.

697 Analysis

698 Cpptraj and Pytraj $^{68}$ are used to analyze all simulation trajectories as per AMBER19 manual ${ }^{69}$, 699 and $\mathrm{VMD}^{70}$ (Visual Molecular Dynamics) is used for visualization.

700 Force curves and analysis. For each unfolding experiment using SMD, the force applied is

701 recorded as a function of the polymer's end-to-end distance. A moving average over 2 - $\AA$ intervals 
702 is calculated to improve signal-to-noise ratios in the force curves. Peak force analysis is done using 703 the SciPy library in Python to characterize the mechanical stability of RHPs and of their 704 homopolymer counterparts. In particular, a feature with peak value greater than $42 \mathrm{pN}$ and a 705 prominence value greater than $28 \mathrm{pN}$ is considered as a peak. For any given sequence, all peaks 706 pooled from the five independent replicates are included in the peak force distribution analysis.

707 Nonequilibrium unfolding work is calculated as the integral of the unfolding force curve evaluated

708 from the initial end-to-end distance to $45 \AA$ for 20 mers and $110 \AA ̊$ for 50 mers, respectively.

709 Dihedral angles. A dihedral angle characterizes bond rotations in a polymer and thus its 710 conformational state. As every four neighboring atoms define a dihedral angle, plotting the 711 dihedral angles along the carbon-carbon backbone of a RHP chain gives a 1D topological 712 fingerprint for that RHP. Dihedral angles are averaged over 2-ns of simulation with standard errors 713 computed, and the absolute value is reported to produce dihedral plots.

714 Contact analysis. Contact maps plotting the intramolecular distances of all possible pairs of 715 monomers in a given chain as a two-dimensional matrix have been extensively used in the 716 literature for protein structural analysis. The internal structure of our RHPs and its evolution upon 717 unfolding is used in an analogous fashion. Three types of contact map analyses are performed in 718 this work based on: $C_{\alpha}$ atoms in the backbone, the center-of-mass of the side-chains, and tail atoms 719 in the side-chains (Figure S2). All contact map data are averaged over 2-ns of simulation 720 trajectories. Contact map data is directly used to determine the number of contacts formed between 721 monomeric residues at a given time (averaged over $2 \mathrm{~ns}$ ). For 50mers, a contact is considered to 722 be established if the monomer-monomer distance, whether it is $C_{\alpha}$-based or side-chain-based, is 723 less than $20 \AA$ A. Excluding non-contact entries in a contact matrix and avoiding double-counting,

724 the number of contacts at a given time is thus given by $n_{\text {contacts }}=\frac{n_{\text {values below threshold }}-n_{\text {diagonal }}}{2}$ where 
$725 n_{\text {values below threshold }}$ is the number of entries in the contact matrix below the threshold and $n_{\text {diagonal }}$

726 is the number of diagonal entries, i.e., 50 for 50 mers.

727 Solvent-accessible surface area. Solvent accessible surface area (SASA) of an RHP is the

728 surface area that is exposed to water molecules as calculated using the linear combination of

729 pairwise overlaps algorithm (LCPO) as implemented in AMBER19 (ref. ${ }^{69}$ ), and all atom

730 contributions from a given RHP molecule are considered for SASA evaluation.

731 Water shell solvation. Solvation data provides information on the solvent-accessible regions of

732 RHPs upon unfolding. The normalized first solvation shell (NFSS) is computed as the number of

733 water molecules in the first solvation shell relative to that in a well-solvated monomer of the same

734 type per Hilburg et al..$^{15}$

735 Statistical analysis

736 ANOVA tests were conducted using Python to ascertain statistical significance $(* p<0.05)$

737 between sampling distributions.

738

739 ASSOCIATED CONTENT

740 Supporting Information.

741 The Supporting Information is available free of charge at xxx.

742 RHP sequence schematics. Atom designations. Heterogeneous conformational sampling in

743 RHPs. Additional stress-relaxation results. Unfolding force curves for all RHPs studied.

744 Characterization of independent unfolding replicates. Snapshots showing the unfolding pathway

745 of a 50mer RHP. OEGMA wrapping: snapshots, contact maps, and monomer-monomer

746 separational distances. Peak force distributions. Additional analysis on non-equilibrium unfolding 
747 work. Effects of backbone topology: unfolding trajectory snapshots, contact map evolutions, and

748 contact reduction curves. Dihedral dynamics. (PDF)

749 Movie of a 50mer RHP unfolding trajectory. (MOV)

750

751 AUTHOR INFORMATION

\section{Corresponding Author}

$753 *$ Alfredo Alexander-Katz - Department of Materials Science and Engineering, Massachusetts

754 Institute of Technology, Cambridge, Massachusetts 02139, United States; Email:

755 aalexand@mit.edu

756 Authors

757 Zexiang Han - orcid.org/0000-0002-8968-0860

758 Shayna L. Hilburg - orcid.org/0000-0003-0825-7698

\section{Funding Sources}

760 This work was supported by the Defense Threat Reduction Agency contract HDTRA11910011.

$761 \quad$ Notes

762 The authors declare no competing financial interest.

\section{ACKNOWLEDGMENTS}

764 Z.H. acknowledges the support from the Imperial-MIT International Research Opportunities

765 Program (IROP) Exchange Program. We are thankful to Ting Xu and Zhiyuan Ruan for insightful 766 discussions. 


\section{REFERENCES}

769 (1) Stirnemann, G.; Kang, S. G.; Zhou, R.; Berne, B. J. How Force Unfolding Differs from Chemical Denaturation. Proc. Natl. Acad. Sci. U. S. A. 2014, 111 (9), 3413-3418. DOI:

(2) Marszalek, P. E.; Lu, H.; Li, H.; Carrion-Vazquez, M.; Oberhauser, A. F.; Schulten, K.; (6757), 100-103. DOI: $10.1038 / 47083$

(3) Lu, H.; Isralewitz, B.; Krammer, A.; Vogel, V.; Schulten, K. Unfolding of Titin Immunoglobulin Domains by Steered Molecular Dynamics Simulation. Biophys. J. 1998, 75 (2), 662-671. DOI: 10.1016/S0006-3495(98)77556-3

(4) Gao, M.; Wilmanns, M.; Schulten, K. Steered Molecular Dynamics Studies of Titin I1 Domain Unfolding. Biophys. J. 2002, 83 (6), 3435-3445. DOI: 10.1016/S00063495(02)75343-5

(5) Paramore, S.; Voth, G. A. Examining the Influence of Linkers and Tertiary Structure in the Forced Unfolding of Multiple-Repeat Spectrin Molecules. Biophys. J. 2006, 91 (9), 34363445. DOI: 10.1529/biophysj.106.091108

6) Lee, G.; Abdi, K.; Jiang, Y.; Michaely, P.; Bennett, V.; Marszalek, P. E. Nanospring Behaviour of Ankyrin Repeats. Nature 2006, 440 (7081), 246-249. DOI: 10.1038/nature04437

(7) Einert, T. R.; Sing, C. E.; Alexander-Katz, A.; Netz, R. R. Conformational Dynamics and Internal Friction in Homopolymer Globules: Equilibrium vs. Non-Equilibrium Simulations. Eur. Phys. J. E 2011, 34 (12). DOI: 10.1140/epje/i2011-11130-8

(8) Alexander-Katz, A.; Wada, H.; Netz, R. R. Internal Friction and Nonequilibrium Unfolding of Polymeric Globules. Phys. Rev. Lett. 2009, 103 (2), 1-4. DOI: 10.1103/PhysRevLett.103.028102

(9) Li, I. T. S.; Walker, G. C. Signature of Hydrophobic Hydration in a Single Polymer. Proc. Natl. Acad. Sci. U. S. A. 2011, 108 (40), 16527-16532. DOI: 10.1073/pnas.1105450108

(10) Chung, J.; Kushner, A. M.; Weisman, A. C.; Guan, Z. Direct Correlation of Single-Molecule Properties with Bulk Mechanical Performance for the Biomimetic Design of Polymers. Nat. Mater. 2014, 13 (11), 1055-1062. DOI: 10.1038/NMAT4090

(11) Hosono, N.; Kushner, A. M.; Chung, J.; Palmans, A. R. A.; Guan, Z.; Meijer, E. W. Forced Unfolding of Single-Chain Polymeric Nanoparticles. J. Am. Chem. Soc. 2015, 137 (21), 
6880-6888. DOI: 10.1021/jacs.5b02967

801 (12) Levy, A.; Feinstein, R.; Diesendruck, C. E. Mechanical Unfolding and Thermal Refolding of Single-Chain Nanoparticles Using Ligand-Metal Bonds. J. Am. Chem. Soc. 2019, 141 (18), 7256-7260. DOI: 10.1021/jacs.9b01960

(13) Geissler, P. L.; Shakhnovich, E. I. Reversible Stretching of Random Heteropolymers. Phys. Rev. E - Stat. Physics, Plasmas, Fluids, Relat. Interdiscip. Top. 2002, 65 (5), 4. DOI: 10.1103/PhysRevE.65.056110

(14) Geissler, P. L.; Shakhnovich, E. I. Mechanical Response of Random Heteropolymers. Macromolecules 2002, 35 (11), 4429-4436. DOI: 10.1021/ma012008e

(15) Hilburg, S. L.; Ruan, Z.; Xu, T.; Alexander-Katz, A. Behavior of Protein-Inspired Synthetic Random Heteropolymers. Macromolecules 2020, 53 (21), 9187-9199. DOI: 10.1021/acs.macromol.0c01886

(17) Neuman, K. C.; Nagy, A. Single-Molecule Force Spectroscopy: Optical Tweezers,

(18) Han, Z; Porter, A. E. In Situ Electron Microscopy of Complex Biological and Nanoscale Systems: Challenges and Opportunities. Front. Nanotechnol. 2020, 2. DOI: 10.3389/fnano.2020.606253

(19) Moerner, W. E. New Directions in Single-Molecule Imaging and Analysis. Proc. Natl. Acad. Sci. U. S. A. 2007, 104 (31), 12596-12602. DOI: 10.1073/pnas.0610081104

(20) Milles, L. F.; Schulten, K.; Gaub, H. E.; Bernardi, R. C. Molecular Mechanism of Extreme Mechanostability in a Pathogen Adhesin. Science 2018, 359 (6383), 1527-1533. DOI: 10.1126/science.aar2094

(21) Bernardi, R. C.; Durner, E.; Schoeler, C.; Malinowska, K. H.; Carvalho, B. G.; Bayer, E. A.; Luthey-Schulten, Z.; Gaub, H. E.; Nash, M. A. Mechanisms of Nanonewton Mechanostability in a Protein Complex Revealed by Molecular Dynamics Simulations and Single-Molecule Force Spectroscopy. J. Am. Chem. Soc. 2019, 141 (37), 14752-14763. DOI: $10.1021 /$ jacs.9b06776

22) Brockwell, D. J.; Paci, E.; Zinober, R. C.; Beddard, G. S.; Olmsted, P. D.; Smith, D. A.; Perham, R. N.; Radford, S. E. Pulling Geometry Defines the Mechanical Resistance of a $\beta$ Sheet Protein. Nat. Struct. Biol. 2003, 10 (9), 731-737. DOI: 10.1038/nsb968 
(23) Deshmukh, S. A.; Li, Z.; Kamath, G.; Suthar, K. J.; Sankaranarayanan, S. K. R. S.; Mancini, D. C. Atomistic Insights into Solvation Dynamics and Conformational Transformation in Thermo-Sensitive and Non-Thermo-Sensitive Oligomers. Polymer (Guildf) 2013, 54 (1), 210-222. DOI: 10.1016/j.polymer.2012.11.009

(24) Imai, S.; Hirai, Y.; Nagao, C.; Sawamoto, M.; Terashima, T. Programmed Self-Assembly Systems of Amphiphilic Random Copolymers into Size-Controlled and Thermoresponsive Micelles in Water. Macromolecules 2018, 51, 398-409. DOI: 10.1021/acs.macromol.7b01918

(25) Xu, Z.; Sun, Y.; Weber, J. K.; Cao, Y.; Wang, W.; Jasinski, D.; Guo, P.; Zhou, R.; Li, J. Directional Mechanical Stability of Bacteriophage $\Phi 29$ Motor's 3WJ-PRNA: Extraordinary Robustness along Portal Axis. Sci. Adv. 2017, 3 (5), 2-10. DOI: 10.1126/sciadv.1601684

(26) Chen, W.; Lou, J.; Hsin, J.; Schulten, K.; Harvey, S. C.; Zhu, C. Molecular Dynamics Simulations of Forced Unbending of Integrin AVB3. PLoS Comput. Biol. 2011, 7 (2). DOI: 10.1371/journal.pcbi.1001086

(27) Bergues-Pupo, A. E.; Arias-Gonzalez, J. R.; Morón, M. C.; Fiasconaro, A.; Falo, F. Role of the Central Cations in the Mechanical Unfolding of DNA and RNA G-Quadruplexes. Nucleic Acids Res. 2015, 43 (15), 7638-7647. DOI: 10.1093/nar/gkv690

(28) Gao, M.; Craig, D.; Lequin, O.; Campbell, I. D.; Vogel, V.; Schulten, K. Structure and Functional Significance of Mechanically Unfolded Fibronectin Type III1 Intermediates. Proc. Natl. Acad. Sci. U. S. A. 2003, 100 (25), 14784-14789. DOI: 10.1073/pnas.2334390100

(29) Guzmán, D. L.; Roland, J. T.; Keer, H.; Kong, Y. P.; Ritz, T.; Yee, A.; Guan, Z. Using Steered Molecular Dynamics Simulations and Single-Molecule Force Spectroscopy to Guide the Rational Design of Biomimetic Modular Polymeric Materials. Polymer (Guildf). 2008, 49 (18), 3892-3901. DOI: 10.1016/j.polymer.2008.06.047.

(30) Ozer, G.; Valecv, E. F.; Quirt, S.; Hernandez, R. Adaptive Steered Molecular Dynamics of the Long-Distance Unfolding of Neuropeptide Y. J. Chem. Theory Comput. 2010, 6 (10), 3026-3038. DOI: $10.1021 / \mathrm{ct} 100320 \mathrm{~g}$

(31) Lu, H.; Schulten, K. Steered Molecular Dynamics Simulations of Force-Induced Protein Domain Unfolding. Proteins Struct. Funct. Genet. 1999, 35 (4), 453-463.

(32) Rico, F.; Gonzalez, L.; Casuso, I.; Puig-Vidal, M.; Scheuring, S. High-Speed Force Spectroscopy Unfolds Titin at the Velocity of Molecular Dynamics Simulations. Science 2013, 342 (6159), 741-743. DOI: 10.1126/science.1239764

(33) Panganiban, B.; Qiao, B.; Jiang, T.; DelRe, C.; Obadia, M. M.; Nguyen, T. D.; Smith, A. A. A.; Hall, A.; Sit, I.; Crosby, M. G.; Dennis, P. B.; Drockenmuller, E.; De La Cruz, M. 
O.; Xu, T. Random Heteropolymers Preserve Protein Function in Foreign Environments. Science 2018, 359 (6381), 1239-1243. DOI: 10.1126/science.aao0335

871

872

873

874

875

876

877

878

879

880

881

882

883

884

885

886

887

888

889

890

891

892

893

894

895

896

897

898

899

900

901

902

(34) Delre, C.; Huang, C.; Li, T.; Dennis, P.; Drockenmuller, E.; Xu, T. Reusable Enzymatic Fiber Mats for Neurotoxin Remediation in Water. ACS Appl. Mater. Interfaces 2018, 10 (51), 44216-44220. DOI: $10.1021 /$ acsami.8b18484

(35) Jiang, T.; Hall, A.; Eres, M.; Hemmatian, Z.; Qiao, B.; Zhou, Y.; Ruan, Z.; Couse, A. D.; Heller, W. T.; Huang, H.; de la Cruz, M. O.; Rolandi, M.; Xu, T. Single-Chain Heteropolymers Transport Protons Selectively and Rapidly. Nature 2020, 577 (7789), 216220. DOI: $10.1038 / \mathrm{s} 41586-019-1881-0$

(36) DelRe, C.; Jiang, Y.; Kang, P.; Kwon, J.; Hall, A.; Jayapurna, I.; Ruan, Z.; Ma, L.; Zolkin, K.; Li, T.; Scown, C. D.; Ritchie, R. O.; Russell, T. P.; Xu, T. Near-Complete Depolymerization of Polyesters with Nano-Dispersed Enzymes. Nature 2021, 592 (7855), 558-563. DOI: 10.1038/s41586-021-03408-3

(37) Mishra, A.; Panwar, A. S.; Chakrabarti, B. Equilibrium Morphologies and Force Extension Behavior for Polymers with Hydrophobic Patches: Role of Quenched Disorder. Macromol. Theory Simulations 2014, 23 (4), 266-278. DOI: 10.1002/mats.201300154

(38) Baul, U.; Chakraborty, D.; Mugnai, M. L.; Straub, J. E.; Thirumalai, D. Sequence Effects on Size, Shape, and Structural Heterogeneity in Intrinsically Disordered Proteins. J. Phys. Chem. B 2019, 123 (16), 3462-3474. DOI: 10.1021/acs.jpcb.9b02575

(39) Verde-Sesto, E.; Arbe, A.; Moreno, A. J.; Cangialosi, D.; Alegría, A.; Colmenero, J.; Pomposo, J. A. Single-Chain Nanoparticles: Opportunities Provided by Internal and External Confinement. Mater. Horizons 2020, 7 (9), 2292-2313. DOI: $10.1039 / \mathrm{d} 0 \mathrm{mh} 00846 \mathrm{j}$

(40) Dobrynin, A. V.; Rubinstein, M. Hydrophobically Modified Polyelectrolytes in Dilute SaltFree Solutions. Macromolecules 2000, 33 (21), 8097-8105. DOI: 10.1021/ma000761m

(41) Müller-Späth, S.; Soranno, A.; Hirschfeld, V.; Hofmann, H.; Rüegger, S.; Reymond, L.; Nettels, D.; Schuler, B. Charge Interactions Can Dominate the Dimensions of Intrinsically Disordered Proteins. Proc. Natl. Acad. Sci. U. S. A. 2010, 107 (33), 14609-14614. DOI: 10.1073/pnas.1001743107

(42) Samatha, P.; Krishnamurti, N. Study on Polymerization of Methyl Methacrylate in WaterSolvent Mixtures. J. Appl. Polym. Sci. 1997, 66 (7), 1419-1423.

(43) Baysal, B. M.; Karasz, F. E. Coil-Globule Collapse in Flexible Macromolecules. Macromol. Theory Simulations 2003, 12 (9), 627-646. DOI: 10.1002/mats.200350028

(44) Hyeon, C.; Thirumalai, D. Mechanical Unfolding of RNA: From Hairpins to Structures with 
Internal Multiloops. Biophys. J. 2007, 92 (3), 731-743. DOI: 10.1529/biophysj.106.093062

904

905

906

907

908

909

910

911

912

913

914

915

916

917

918

919

920

921

922

923

924

925

926

927

928

929

930

931

932

933

934

935

936

(45) Liu, M.; Leroux, J. C.; Gauthier, M. A. Conformation-Function Relationships for the CombShaped Polymer POEGMA. Prog. Polym. Sci. 2015, 48, 111-121. DOI: 10.1016/j.progpolymsci.2015.03.001

(46) Yadavalli, N. S.; Borodinov, N.; Choudhury, C. K.; Quiñones-Ruiz, T.; Laradji, A. M.; Tu, S.; Lednev, I. K.; Kuksenok, O.; Luzinov, I.; Minko, S. Thermal Stabilization of Enzymes with Molecular Brushes. ACS Catal. 2017, 7 (12), 8675-8684. DOI: 10.1021/acscatal.7b03138

(47) Lee, P.; Towslee, J.; Maia, J.; Pokorski, J. PEGylation to Improve Protein Stability during Melt Processing. Macromol. Biosci. 2015, $15 \quad$ (10), 1332-1337. DOI: 10.1002/mabi.201500143

(48) Kaupbayeva, B.; Boye, S.; Munasinghe, A.; Murata, H.; Matyjaszewski, K.; Lederer, A.; Colina, C. M.; Russell, A. J. Molecular Dynamics-Guided Design of a Functional ProteinATRP Conjugate That Eliminates Protein-Protein Interactions. Bioconjug. Chem. 2021. DOI: acs.bioconjchem.1c00098

(49) Debenedictis, E. P.; Hamed, E.; Keten, S. Mechanical Reinforcement of Proteins with Polymer Conjugation. ACS Nano 2016, 10 (2), 2259-2267. DOI: 10.1021/acsnano.5b06917

(50) Lawrence, P. B.; Price, J. L. How PEGylation Influences Protein Conformational Stability. Curr. Opin. Chem. Biol. 2016, 34, 88-94. DOI: 10.1016/j.cbpa.2016.08.006

(51) Wilding, K. M.; Smith, A. K.; Wilkerson, J. W.; Bush, D. B.; Knotts, T. A.; Bundy, B. C. The Locational Impact of Site-Specific PEGylation: Streamlined Screening with Cell-Free Protein Expression and Coarse-Grain Simulation. ACS Synth. Biol. 2018, 7 (2), 510-521. DOI: $10.1021 /$ acssynbio.7b00316

(52) Bryant, Z.; Pande, V. S.; Rokhsar, D. S. Mechanical Unfolding of a $\beta$-Hairpin Using Molecular Dynamics. Biophys. J. 2000, 78 (2), 584-589. DOI: 10.1016/S00063495(00)76618-5

(53) Hyeon, C.; Dima, R. I.; Thirumalai, D. Pathways and Kinetic Barriers in Mechanical Unfolding and Refolding of RNA and Proteins. Structure 2006, 14 (11), 1633-1645. DOI: 10.1016/j.str.2006.09.002

(54) Rodriguez-Larrea, D.; Ibarra-Molero, B.; Sanchez-Ruiz, J. M. Energetic and Structural Consequences of Desolvation/Solvation Barriers to Protein Folding/Unfolding Assessed from Experimental Unfolding Rates. Biophys. J. 2006, 91 (5), 48-50. DOI: 10.1529/biophysj.106.087932

(55) Soranno, A.; Buchli, B.; Nettels, D.; Cheng, R. R.; Müller-Späth, S.; Pfeil, S. H.; Hoffmann, 
A.; Lipman, E. A.; Makarov, D. E.; Schuler, B. Quantifying Internal Friction in Unfolded and Intrinsically Disordered Proteins with Single-Molecule Spectroscopy. Proc. Natl. Acad. Sci. U. S. A. 2012, 109 (44), 17800-17806. DOI: 10.1073/pnas.1117368109

(56) Zheng, W.; Hofmann, H.; Schuler, B.; Best, R. B. Origin of Internal Friction in Disordered

(57) Brendel, V.; Bucher, P.; Nourbakhsh, I. R.; Blaisdell, B. E.; Karlin, S. Methods and

(58) Scalvini, B.; Sheikhhassani, V.; Woodard, J.; Aupič, J.; Dame, R. T.; Jerala, R.; Mashaghi,

(59) Heidari, M.; Heidari, M.; Schiessel, H.; Mashaghi, A. Circuit Topology Analysis of

(60) Shank, E. A.; Cecconi, C.; Dill, J. W.; Marqusee, S.; Bustamante, C. The Folding

(61) Paci, E.; Karplus, M. Unfolding Proteins by External Forces and Temperature: The A. Topology of Folded Molecular Chains: From Single Biomolecules to Engineered Origami. Trends Chem. 2020, 2 (7), 609-622. DOI: 10.1016/j.trechm.2020.04.009 Polymer Folding Reactions. ACS Cent. Sci. 2020, 6 (6), 839-847. DOI: 10.1021/acscentsci.0c00308 Cooperativity of a Protein Is Controlled by Its Chain Topology. Nature 2010, 465 (7298), 637-640. DOI: 10.1038/nature09021 Importance of Topology and Energetics. Proc. Natl. Acad. Sci. U. S. A. 2000, 97 (12), 65216526. DOI: $10.1073 /$ pnas. 100124597

(62) Klimov, D. K.; Thirumalai, D. Native Topology Determines Force-Induced Unfolding Pathways in Globular Proteins. Proc. Natl. Acad. Sci. U. S. A. 2000, 97 (13), 7254-7259. DOI: $10.1073 /$ pnas.97.13.7254

(63) Polotsky, A. A.; Charlaganov, M. I.; Leermakers, F. A. M.; Daoud, M.; Borisov, O. V.; Birshtein, T. M. Mechanical Unfolding of a Homopolymer Globule Studied by SelfConsistent Field Modeling. Macromolecules 2009, 42 (14), 5360-5371. DOI: $10.1021 / \mathrm{ma9} 904742$

(64) Ma, C. D.; Wang, C.; Acevedo-Vélez, C.; Gellman, S. H.; Abbott, N. L. Modulation of Hydrophobic Interactions by Proximally Immobilized Ions. Nature 2015, 517 (7534), 347350. DOI: $10.1038 /$ nature 14018

(65) Huang, K.; Gast, S.; Ma, C. D.; Abbott, N. L.; Szlufarska, I. Comparison between Free and Immobilized Ion Effects on Hydrophobic Interactions: A Molecular Dynamics Study. $J$. Phys. Chem. B 2015, 119 (41), 13152-13159. DOI: 10.1021/acs.jpcb.5b05220 
(66) Perez-Baena, I.; Asenjo-Sanz, I.; Arbe, A.; Moreno, A. J.; Lo Verso, F.; Colmenero, J.; Pomposo, J. A. Efficient Route to Compact Single-Chain Nanoparticles: Photoactivated Synthesis via Thiol-Yne Coupling Reaction. Macromolecules 2014, 47 (23), 8270-8280. DOI: $10.1021 / \mathrm{ma} 5017133$

(67) Formanek, M.; Moreno, A. J. Effects of Precursor Topology and Synthesis under Crowding Conditions on the Structure of Single-Chain Polymer Nanoparticles. Soft Matter 2017, 13 (37), 6430-6438. DOI: $10.1039 / \mathrm{c} 7 \mathrm{sm} 01547 \mathrm{j}$

(68) Roe, D. R.; Cheatham, T. E. PTRAJ and CPPTRAJ: Software for Processing and Analysis of Molecular Dynamics Trajectory Data. J. Chem. Theory Comput. 2013. DOI: $10.1021 / \mathrm{ct} 400341 \mathrm{p}$

(69) D.A. Case, I.Y. Ben-Shalom, S.R. Brozell, D.S. Cerutti, T.E. Cheatham, III, V.W.D. Cruzeiro, T.A. Darden, R.E. Duke, D. Ghoreishi, G. Giambasu, T. Giese, M.K. Gilson, H. Gohlke, A.W. Goetz, D. Greene, R Harris, N. Homeyer, Y. Huang, S. Izadi, A. Kovalenko, R. Krasny, T. Kurtzman, T.S. Lee, S. LeGrand, P. Li, C. Lin, J. Liu, T. Luchko, R. Luo, V. Man, D.J. Mermelstein, K.M. Merz, Y. Miao, G. Monard, C. Nguyen, H. Nguyen, A. Onufriev, F. Pan, R. Qi, D.R. Roe, A. Roitberg, C. Sagui, S. Schott-Verdugo, J. Shen, C.L. Simmerling, J. Smith, J. Swails, R.C. Walker, J. Wang, H. Wei, L. Wilson, R.M. Wolf, X. $\mathrm{Wu}$, L. Xiao, Y. Xiong, D.M. York and P.A. Kollman. AMBER 2019, University of California, San Francisco. 2019.

(70) Humphrey, W.; Dalke, A.; Schulten, K. Sartorius Products. J. Mol. Graph. 1996, 14 (October 1995), 33-38. 